\title{
Nuclear Wobbling Motion and Electromagnetic Transitions
}

\author{
Yoshifumi R. Shimizu and Masayuki Matsuzaki*) \\ Department of Physics, Kyushu University 33, Fukuoka 812, Japan \\ *) Department of Physics, Fukuoka University of Education, \\ Munakata, Fukuoka 811-41, Japan
}

\begin{abstract}
The nuclear wobbling motion is studied from a microscopic viewpoint. It is shown that the expressions not only of the excitation energy but also of the electromagnetic transition rate in the microscopic RPA framework can be cast into the corresponding forms of the macroscopic rotor model. Criteria to identify the rotational band associated with the wobbling motion are given, based on which examples of realistic calculations are investigated and some theoretical predictions are presented.
\end{abstract}

\section{$\S 1$ Introduction}

The recent advent of new generation crystal ball detectors has been opening a great possibility to explore a new area of the high-spin physics. There are many interesting subjects which are waiting to be studied. Among them, we would like to concentrate, in this paper, upon the nuclear wobbling motion, which is one of the "exotic" rotational motions in the sense that the axis of rotation does not coincide with any of the inertia axes of deformation.

The nuclear wobbling motion has been considered by analogy with the spinning motions of asymmetric top (classical rigid-body), where perturbations are superimposed on the main rotation around one of the principal axes with the largest moment of inertia. When quantized, the energy spectra in the energy versus angular momentum plane, which are nothing but those of the well-known macroscopic triaxial rotor model (Davydov model), are classified into two groups of rotational bands, i.e., the "horizontal" and "vertical" sequences corresponding asymptotically to the Regge trajectories associated with the largest and the smallest moment of inertia. In the high-spin limit, ${ }^{1)}$ the physical meaning of those two sequences appears to be more transparent by introducing an elementary excitation of the "wobbling phonon" mode. The horizontal sequences parallel to the yrast line are rotational bands in which zero, one, and two, etc, wobbling phonons are excited in each intrinsic state, 
while the vertical sequences starting from each yrast state consist of the multiple wobbling phonon bands. In fact, the $E 2$ transition rates in the former $(|\Delta I|=2)$ are larger than that in the latter $(|\Delta I|=1)$ by an order of $1 / I$ in the high-spin limit. ${ }^{1,2)}$

It is very interesting to ask whether such an "exotic" rotational motion is realized as a collective motion in atomic nuclei, because it directly reflects the three-dimensional nature of rotational motions. It should, however, be noticed that the concept of the threedimensional rotation is meaningful only when the motion of angular momentum vector is traced in the "intrinsic" or the "body-fixed" frame, where the macroscopic rotor model is formulated. The very definition, however, of such a coordinate frame is highly nontrivial from the microscopic point of view in the general framework of the nuclear many-body theory. $^{3-6)}$

Detailed experimental investigation is only possible in the discrete line spectroscopy so that the one or two phonons excited horizontal sequences are the most promising targets for the study. For such rotational bands near the yrast line, the small amplitude approximation to the wobbling mode may be allowed. Then the fully microscopic formulation is possible $^{2,7-10)}$ in terms of the random phase approximation (RPA) on top of the cranked mean-field theory, and the analogy to the macroscopic rotor model becomes transparent. Thus we mainly concentrate, in this paper, on the yrast and the first excited wobbling bands and investigate the possible consequences predicted from the RPA theory ${ }^{2)}$ in realistic nuclei. In the course of the investigation it will be shown that not only the energy spectra but also the interband $(\Delta I= \pm 1)$ electromagnetic transition probabilities can also be expressed in the same way as in the macroscopic rotor model in terms of the microscopically defined "effective" moments of inertia, ${ }^{2)}$ which are introduced for the wobbling eigen frequency.

The paper is organized as follows: the $E 2$ transitions in the RPA theory is reviewed and is applied to the well-known $\gamma$-vibrational bands in $\S 2$. Some basic ingredients in the macroscopic rotor model, especially the expressions for the electromagnetic transition rates, are reviewed in $\S 3$ for the completeness. The microscopic formalism is presented in $\S 4$, while some examples of realistic calculations are studied in $\S 5$. Main results are summarized in $\S 6$. Preliminary results of some part of the present work has already been 
reported in Refs.. ${ }^{11-13)}$

\section{$\S 2$ E2 Transitions for the $\gamma$-vibrational band}

The RPA theory on top of the cranked mean-field approach, which is suitable for the high-spin states, was first developed in Ref. ${ }^{14)}$ and applied to the high-spin $\beta$ - and $\gamma$ vibrational bands. $^{15,16)}$ The extension to the odd nuclei, with special attention to how the electromagnetic transition rates should be calculated, has been done in Ref.. ${ }^{17)}$

The RPA treatment of the vibrational excitations ${ }^{14)}$ is based on the boson expansion theory; the lowest order vacuum states, on which the RPA modes are excited, are described by the static mean-field theory uniformly rotating around one of the inertia axes (one dimensional cranking states). Combined with the $1 / I$ expansion technique, the matrix element of the electromagnetic transition with multipolarity $\lambda$ is expressed in the simple form;

$$
\mathcal{M}(i \rightarrow f ; \Delta I) \approx\left\langle f\left|Q_{\lambda \mu=\Delta I}^{(E)}\right| i\right\rangle
$$

where $\Delta I=I_{f}-I_{i}$ and the superscript $(E)$ means the electric part of the transition operator $Q_{\lambda \mu}$. It should be stressed that the components of $Q_{\lambda \mu}^{(E)}$ are defined with respect to the rotation (cranking) axis.

In the formula above only the lowest order in $1 / I$ is retained. Although we are mainly concerned with the high-spin limit and consider only in the order of eq.(2.1) in the following sections, it is worth while mentioning that the formula is applicable at low-spins if the the geometry of the angular momentum vector is properly taken into account. A good example is the $M 1$ transition in odd nuclei at relatively low angular momenta. ${ }^{18)}$ It is, however, noticed that the idea is more general and is based on the observation that the most important part of the spin-dependence, which comes from the dynamics not from the kinematics of the angular momentum algebra (the Clebsch-Gordan coefficients), is contained in the right hand side through the spin-dependent change of cranked wave functions. In order to see this is really the case and to show the reliability of the formula (2.1), we compare, in the remaining part of this section, the results of the RPA calculations for the low-spin $\gamma$-vibrational band ${ }^{16)}$ in even-even nuclei with the experimental data.

The quantities to be investigated are the $E 2$ transitions between the ground state band 
and the $\gamma$-vibrational band. Therefore, the initial and the final state in eq.(2.1) are

$$
|i\rangle=\hat{X}_{\gamma}^{( \pm) \dagger}\left|\omega_{\text {rot }}\right\rangle, \quad|f\rangle=\left|\omega_{\text {rot }}\right\rangle
$$

where $\left|\omega_{\text {rot }}\right\rangle$ is cranked mean-field approximation of the yrast states with rotational frequency $\omega_{\text {rot }}$, and $\hat{X}_{\gamma}^{( \pm) \dagger}$ is the creation operator of the RPA eigen mode at the corresponding frequency, which smoothly continues to the $\gamma$-vibrational solution at zero-frequency. The superscript $( \pm)^{16)}$ denotes the signature quantum number carried by the RPA mode, so that, for example, the $(+)$-band represents the even-spin member and the $(-)$-band the odd-spin if the vacuum $\left|\omega_{\text {rot }}\right\rangle$ has zero signature. There are five kinds of transitions associated with $\Delta I=0, \pm 1, \pm 2$, and the $B(E 2)$-values of these transitions are calculated by means of the electric (i.e. proton) part of the operators, ${ }^{17}$ )

$$
\begin{gathered}
Q_{20}=\frac{1}{2} Q_{0}^{(+)}+\frac{\sqrt{3}}{2} Q_{2}^{(+)} \\
Q_{2 \pm 1}=\frac{i}{\sqrt{2}}\left(Q_{1}^{(-)} \pm Q_{2}^{(-)}\right) \\
Q_{2 \pm 2}=-\frac{1}{\sqrt{2}}\left(\frac{\sqrt{3}}{2} Q_{0}^{(+)}+\frac{1}{2} Q_{2}^{(+)} \pm Q_{1}^{(+)}\right),
\end{gathered}
$$

where $Q_{K}^{( \pm)}(K=0,1,2)$ are the signature coupled quadrupole operators with the $z$-axis as the quantization axis, while the rotation axis is chosen to be the $x$-axis as usual.

Among the five transition amplitudes between the $\gamma$ and the ground state band,

$$
t\left[\left(Q_{K}^{( \pm)}\right)\right] \equiv\left\langle f\left|Q_{K}^{( \pm)}\right| i\right\rangle=\left\langle\omega_{\text {rot }}\left|\left[Q_{K}^{( \pm)}, \hat{X}_{\gamma}^{( \pm) \dagger}\right]\right| \omega_{\text {rot }}\right\rangle=\left\langle\left[Q_{K}^{( \pm)}, \hat{X}_{\gamma}^{( \pm) \dagger}\right]\right\rangle
$$

only the $K=2$ components are non-zero at $\omega_{\text {rot }}=0$ from the selection rule because the ground state under consideration is axially symmetric. Moreover, the electric part of them are of the form

$$
t\left[\left(Q_{K}^{( \pm)}\right)^{(E)}\right]=\mathcal{Q}_{\gamma}\left(\delta_{K 2}+\delta_{K 1} a_{\gamma} \omega_{\text {rot }}\right)+O\left(\omega_{\text {rot }}^{2}\right)
$$

in the low frequency limit. As it is mentioned we have to include the effect of ClebschGordan coefficients at the low-spin. In this case $\left\langle I_{i} 22-2 \mid I_{f} 0\right\rangle$ is necessary, which has the asymptotic values in the large $I$ limit;

$$
\left|\left\langle I_{i} 22-2 \mid I_{f} 0\right\rangle\right| \approx\left\{\begin{array}{cl}
\sqrt{6} / 4 & \Delta I=0, \\
1 / 2 & \Delta I= \pm 1, \\
1 / 4 & \Delta I= \pm 2 .
\end{array}\right.
$$


Combining with eqs.(2.3) and (2.6) we have the expression of the $B(E 2) \equiv|\mathcal{M}(i \rightarrow f)|^{2}$, which is valid in the low-spin limit,

$$
\frac{B(E 2)_{\gamma \rightarrow g}^{\Delta I}}{\left\langle I_{i} 22-2 \mid I_{f} 0\right\rangle^{2}}=\left|\sqrt{2} \mathcal{Q}_{\gamma}\right|^{2}\left(1+a_{\gamma} \Delta I \omega_{\text {rot }}+O\left(\omega_{\text {rot }}^{2}\right)\right)^{2},
$$

where eq.(2.5) is inserted. Using again the asymptotic relation and the fact that the rotational frequency can be related to the angular momentum through the moment of inertia $\mathcal{J}$,

$$
I_{f}\left(I_{f}+1\right)-I_{i}\left(I_{i}+1\right) \approx 2 I \Delta I, \quad \hbar \omega_{\mathrm{rot}} \approx I / \mathcal{J},
$$

where $I \equiv\left(I_{f}+I_{i}\right) / 2$, we finally find

$$
\frac{\left[B(E 2)_{\gamma \rightarrow g}^{\Delta I}\right]^{\frac{1}{2}}}{\left\langle I_{i} 22-2 \mid I_{f} 0\right\rangle} \approx \mathcal{Q}_{t}\left(1+q\left[I_{f}\left(I_{f}+1\right)-I_{i}\left(I_{i}+1\right)\right]\right),
$$

in the first order, which is nothing but the formula discussed as the generalized intensity relation in Ref., ${ }^{19)}$ where

$$
\mathcal{Q}_{t} \equiv\left|\sqrt{2} \mathcal{Q}_{\gamma}\right|, \quad q \equiv a_{\gamma} / 2 \hbar \mathcal{J}
$$

It should be emphasized that the parameters $\mathcal{Q}_{t}$ and $q$ are now calculated microscopically by means of the RPA theory at the finite rotational frequency.

An example of the RPA amplitudes of the electric quadrupole transition operators, eq.(2.4), as functions of the rotational frequency is shown in Fig.1 for a typical welldeformed nuclei, ${ }^{164} \mathrm{Er}$. The procedure of calculation is the same as Refs. ${ }^{16,17)}$ except that the difference of the oscillator frequency between neutrons and protons in the Nilsson potential and of the oscillator length in the quadrupole residual interaction ${ }^{20,21)}$ are properly treated. $^{*}$ Namely, the residual interactions of the monopole pairing and the quadrupole interactions are used, the strengths of which are determined so as to reproduce the evenodd mass difference, and the excitation energies of the $\beta, \gamma$-vibrations $(K=0,2)$ and the

*) In Ref. ${ }^{16)}$ the RPA amplitudes of the $\gamma$-vibration for the mass quadrupole operators were shown. For the collective solutions the proper treatment of neutron and proton oscillator lengths makes the transition amplitudes of the electric operators approximately about $Z / A$ of those of the mass operators, just as in the case of the static quadrupole moments, i.e. the mean values of the mass and electric quadrupole operators, see eq.(4.32) and discussions at the end of $\S 4$. 
zero-frequency (Nambu-Goldstone) mode $(K=1)$, at $\omega_{\text {rot }}=0$, respectively. After fixing the force strengths at $\omega_{\text {rot }}=0$, the pairing selfconsistent calculations are performed as functions of the rotational frequency, but the deformation parameters are fixed at the values deduced from the experiments, ${ }^{22)}$ for simplicity. Increasing the rotational frequency, the quasiparticle alignments generally occur. In order to identify individual rotational bands with the same internal structure, are used the diabatic quasiparticle orbits, ${ }^{16)}$ specifically the diabatic basis constructed by the method of the $\omega_{\text {rot }}$-expansion up to the third order. ${ }^{17}$ The model space of the Nilsson orbits are chosen as $N_{\text {osc }}=4-6$ for neutrons and $=3-5$ for protons, which reproduces the transition amplitude very well without using any kind of the effective charge (see below).

From the figure it is seen that the amplitudes follow the low frequency behaviours of eq.(2.5) and the parameters, $\mathcal{Q}_{\gamma}$ and $a_{\gamma}$, are easily extracted. The values thus obtained for some arbitrarily chosen rare earth nuclei are summarized in Table 1, where the parameters in eq.(2.9), $\mathcal{Q}_{t}$ and $q$, are also included and compared with the available experimental data. Note that the moments of inertia for the ground state band and the $\gamma$-vibrational band are the same in the low-spin limit within the RPA theory. It is not the case in experimental data, however. This is because the difference of the moments of inertia results from the higher order couplings between the vibrational and rotational motions, which are not taken into account in the RPA. Therefore two values of $q$ which are obtained by using two choices of experimentally determined $\mathcal{J}=\mathcal{J}_{g}$ and $\mathcal{J}=\mathcal{J}_{\gamma}$ are included in the table.

The absolute values of the amplitudes are well reproduced in our calculations. However, the agreement might not be taken so seriously because the resultant RPA amplitude depends on the size of the adopted model space. It is known that the calculation with full model space usually overestimates the experimental values in the simple monopole pairing plus the quadrupole interaction model. We will not discuss this point further in this paper.

More important is that not only the sign but also absolute values of the $q$ parameter are well accounted for in the present RPA theory, which is calculated from the ratio of the transition amplitudes and therefore more reliable than the amplitudes themselves. This clearly shows the rotation-induced change of the microscopic structure of the vibrational motion, i.e. the $K$-mixing of the transition amplitudes, see eq.(2.5), are correctly described 
in the RPA theory. In Fig.1 the appreciable deviations from the lowest order relations, the order of $O\left(\omega_{\text {rot }}^{2}\right)$, are predicted in the region of $\hbar \omega_{\text {rot }} \gtrsim 0.15 \mathrm{MeV}$, for example, the amount of the reduction of the $I_{\gamma} \rightarrow(I-1)_{g}$ transition rates are larger than that of the $I_{\gamma} \rightarrow(I-2)_{g}$ transitions (see eq.(2.3)). We should, however, be careful to draw a definite conclusion: at these moderate spins other higher order effects in $1 / I$ neglected in, e.g., eqs.(2.6), (2.8), are of the same order, and then all the higher order terms should be consistently calculated, which is out of the scope of the present calculations.

\section{$\S 3$ Macroscopic Rotor Model}

The nuclear wobbling motion was originally predicted as a collective motion in the macroscopic rotor model. ${ }^{1)}$ In order to see the characteristic features and to compare with the microscopic model, the energy spectra and the $E 2$ and $M 1$ transition rates in the high-spin limit are summarized in this section.

The hamiltonian of the rotor model is written in terms of the three moments of inertia around the principal axes, $\mathcal{J}_{x}, \mathcal{J}_{y}$ and $\mathcal{J}_{z}$ :

$$
H_{R}=\frac{I_{x}^{2}}{2 \mathcal{J}_{x}}+\frac{I_{y}^{2}}{2 \mathcal{J}_{y}}+\frac{I_{z}^{2}}{2 \mathcal{J}_{z}}
$$

where the angular momentum operators here are components with respect to the body-fixed frame. The energy spectrum of the above hamiltonian is well known: it is specified by two quantum numbers and they are given in the large $I$ limit explicitly by

$$
E_{R}\left(I, n_{w}\right)=\frac{I(I+1)}{2 \mathcal{J}_{x}}+\hbar \omega_{w}(I)\left(n_{w}+\frac{1}{2}\right),
$$

where $\mathcal{J}_{x}$ is assumed to be the largest and the main rotation occurs around the $x$-axis. The wobbling frequency is determined by the well-known formula, ${ }^{1,23)}$

$$
\hbar \omega_{w}=I \sqrt{W_{y} W_{z}}=\hbar \omega_{\mathrm{rot}} \sqrt{\frac{\left(\mathcal{J}_{x}-\mathcal{J}_{y}\right)\left(\mathcal{J}_{x}-\mathcal{J}_{z}\right)}{\mathcal{J}_{y} \mathcal{J}_{z}}}
$$

where

$$
\hbar \omega_{\text {rot }} \equiv I / \mathcal{J}_{x}
$$

corresponds to the rotational frequency around the main rotation axis and the quantities,

$$
W_{y} \equiv 1 / \mathcal{J}_{z}-1 / \mathcal{J}_{x}, \quad W_{z} \equiv 1 / \mathcal{J}_{y}-1 / \mathcal{J}_{x}
$$


are introduced. The integer $n_{w}=0,1,2, \ldots$ in eq.(3.2) is the wobbling phonon number excited on the yrast states. Then the horizontal and vertical sequences mentioned in $\S 1$ are precisely

$$
E_{|\Delta I|=2}^{(\text {hor })}(I)=E_{R}\left(I, n_{w}\right), \quad n_{w}=0,1,2, \ldots
$$

with $n_{w}$ specifying the yrast, yrare,... bands, and

$$
E_{|\Delta I|=1}^{(\text {ver })}(I)=E_{R}\left(I, n_{w}=I-K\right), \quad K=K_{1}, K_{2}, K_{3}, \ldots
$$

with $K$ specifying the band head spin. Note that the number of phonons are changed in the vertical sequence, while it is unchanged in the horizontal one. These two classifications correspond to the band structure connected by the $E 2$ transitions with $\Delta I= \pm 2$ and $\Delta I= \pm 1$, respectively, with the transition energies, in the lowest order in $1 / I$,

$$
\begin{gathered}
E_{\gamma}^{(\text {hor })}=\mp 2 \hbar \omega_{\text {rot }}, \quad(\Delta I= \pm 2) . \\
E_{\gamma}^{(\text {ver })}=\hbar \omega_{w} \mp \hbar \omega_{\text {rot }}, \quad(\Delta I= \pm 1) .
\end{gathered}
$$

The $E 2$ transition operator in the rotor model is derived by the basic assumption that the quadrupole tensor of the rotor is diagonal in the body-fixed frame; thus

$$
Q_{2 \mu}^{(E)}=e\left(\frac{Z}{A}\right) R^{2}\left\{\frac{\alpha_{y}+\alpha_{z}}{\sqrt{3}} D_{\mu, 0}^{(2)}+\frac{\alpha_{y}-\alpha_{z}}{\sqrt{2}}\left(D_{\mu, 2}^{(2)}+D_{\mu,-2}^{(2)}\right)\right\}
$$

and then the transition rates is given, again within the large $I$ approximation, by

$$
\begin{gathered}
B(E 2)_{\Delta I= \pm 2}^{(\text {hor })} \approx\left(e \frac{Z}{A}\right)^{2} \frac{1}{2} R^{4}\left(\alpha_{y}-\alpha_{z}\right)^{2} \\
B(E 2)_{\Delta I= \pm 1}^{(\mathrm{ver})} \approx\left(e \frac{Z}{A}\right)^{2} \frac{n_{w}}{I} R^{4}\left(\alpha_{y}\left(\frac{W_{z}}{W_{y}}\right)^{\frac{1}{4}} \mp \alpha_{z}\left(\frac{W_{y}}{W_{z}}\right)^{\frac{1}{4}}\right)^{2} .
\end{gathered}
$$

Here the deformation parameters $\left(\alpha_{y}, \alpha_{z}\right)$ are introduced through the static quadrupole moments for later convenience;

$$
\begin{aligned}
R^{2} \alpha_{y} & \equiv \frac{1}{2} \sqrt{\frac{15}{4 \pi}} \int\left(x^{2}-z^{2}\right) \rho(\boldsymbol{r}) d \boldsymbol{r}=\left\langle\frac{1}{2} Q_{2}^{(+)}-\frac{\sqrt{3}}{2} Q_{0}^{(+)}\right\rangle, \\
R^{2} \alpha_{z} & \equiv \frac{1}{2} \sqrt{\frac{15}{4 \pi}} \int\left(x^{2}-y^{2}\right) \rho(\boldsymbol{r}) d \boldsymbol{r}=\left\langle Q_{2}^{(+)}\right\rangle,
\end{aligned}
$$


in obvious notations. These parameters, $\left(\alpha_{y}, \alpha_{z}\right)$, can be related to the usual " $\left(\epsilon_{2}, \gamma\right)$ "-like parametrization $\left(\epsilon_{2}^{*}, \gamma^{*}\right)^{*)}$ which are defined by

$$
\int x_{k}^{2} \rho(\boldsymbol{r}) d \boldsymbol{r} \equiv \frac{1}{3} R^{2}\left(1+\frac{4}{3} \epsilon_{2}^{*} \cos \left(\gamma^{*}+\frac{2 \pi}{3} k\right)\right), \quad(k=1,2,3 \equiv x, y, z)
$$

through

$$
\alpha_{y}=\sqrt{\frac{5}{9 \pi}} \epsilon_{2}^{*} \sin \left(\gamma^{*}+\frac{4 \pi}{3}\right), \quad \alpha_{z}=-\sqrt{\frac{5}{9 \pi}} \epsilon_{2}^{*} \sin \gamma^{*},
$$

so that they represent the static triaxiality around the corresponding $y, z$-axes. In the same way $\left(\alpha_{y}-\alpha_{z}\right)$ is proportional to the static moments around the main rotation $(x)$ axis and then eq.(3.7a) simply denotes the usual stretched $E 2$ transitions with respect to the $x$-axis. If we consider the lowest excited band, the 1-phonon wobbling band, then $n_{w}=1$ in eq. $(3.7 \mathrm{~b})$.

In the same framework $M 1$ transitions can be considered if an appropriate magnetic property of the rotor is assumed. According to Ref., ${ }^{24)}$ we assume that each component of the magnetic moment vector in the body-fixed frame is proportional to that of the angular momentum,

$$
m_{i}=g_{i} I_{i} \quad(i=x, y, z),
$$

where $g_{i}(i=x, y, z)$ denotes the $\mathrm{g}$-factor with respect to the $i$-th axis in the body-fixed frame. Then the $M 1$ transition operator is derived as ${ }^{\dagger)}$

$$
\begin{aligned}
\mu_{1 \mu}=\sqrt{\frac{3}{4 \pi}} \mu_{N}\left\{g_{x} I_{\mu}^{(l a b)}\right. & +\frac{1}{2}\left(g_{y}+g_{z}-2 g_{x}\right)\left(I_{+1} D_{\mu,+1}^{(1)}+I_{-1} D_{\mu,-1}^{(1)}\right), \\
& \left.-\frac{1}{2}\left(g_{y}-g_{z}\right)\left(I_{-1} D_{\mu,+1}^{(1)}+I_{+1} D_{\mu,-1}^{(1)}\right)\right\}
\end{aligned}
$$

where $\mu_{N}$ is the nuclear magneton and $I_{\mu}^{(l a b)}$ means the angular momentum operator in the laboratory frame so that the first term in the curly bracket does not contribute to the transitions. The $B(M 1)$ is evaluated as in the same way as $B(E 2)$;

$$
B(M 1)_{\Delta I= \pm 1}^{(\mathrm{ver})} \approx\left(\frac{3}{4 \pi} \mu_{N}^{2}\right) n_{w} \frac{I}{4}\left(\left(g_{y}-g_{x}\right)\left(\frac{W_{y}}{W_{z}}\right)^{\frac{1}{4}} \mp\left(g_{z}-g_{x}\right)\left(\frac{W_{z}}{W_{y}}\right)^{\frac{1}{4}}\right)^{2} .
$$

*) These parameters coincide with the original ones only within the first order in $\epsilon_{2}$ because the original ones are defined with respect to the anisotropic harmonic oscillator frequencies. The Lund convention for the sign of triaxiality parameter $\gamma$ is used throughout in this paper.

$\dagger$ ) The $M 1$ operator used in Ref. ${ }^{24)}$ is different from eq.(3.12), which, we think, is incorrect. The resultant $B(M 1)$ is also different. 
Note that $B(E 2)_{\Delta I= \pm 1}^{(\text {ver })} \propto 1 / I$ and $B(M 1)_{\Delta I= \pm 1}^{(\text {ver })} \propto I$ while $B(E 2)_{\Delta I= \pm 2}^{(\text {hor })}$ does not depend on $I$ (up to the leading order in $1 / I$ ), if all the parameters, the quadrupole deformations, $\alpha_{y}$ and $\alpha_{z}$, the moments of inertia, $\mathcal{J}_{x}, \mathcal{J}_{y}$ and $\mathcal{J}_{z}$, and the g-factors, $g_{x}, g_{y}$ and $g_{z}$, are constants against $I$. Actually, all these parameters are not independent and, for example, the quadrupole deformations completely determine the moments of inertia in the original rigid-body model. However, if one wants to apply the model to realistic nuclei, one should consider that all the parameters depends smoothly on the angular momentum, and the moments of inertia do not necessarily take the rigid-body values. As will be discussed in the following sections, the microscopically calculated $\mathcal{J}_{x}, \mathcal{J}_{y}$ and $\mathcal{J}_{z}$ change as functions of the rotational frequency and the relationship between them are far from that of the rigid-body. $^{25)}$

It is worth mentioning that the axially symmetric limit should be taken with great care. There are two kind of limits: the "collective rotation" limit, where $\gamma=0^{\circ},-60^{\circ}$, or equivalently either $\alpha_{z}=0$ or $\alpha_{y}=0$, and the "non-collective rotation" limit where $\gamma=60^{\circ},-120^{\circ}$, or $\alpha_{y}=\alpha_{z}$. In the former limit, it is easy to see that there are no definite limiting expressions for the wobbling energy and the $\Delta I= \pm 1 B(E 2)$ and $B(M 1)$, as long as the detailed limiting behaviours of three moment of inertia are not specified. In contrast, one can always argue the latter limit, where, of course, $\mathcal{J}_{y}=\mathcal{J}_{z}$ so that $W_{y}=W_{z}$. Then, from eq.(3.7), the $\Delta I=+1$ vertical transition as well as the $\Delta I= \pm 2$ horizontal transitions are prohibited. In fact, in this axially symmetric limit of the "non-collective rotation", the horizontal rotational sequences disappear and only the band heads with $\left\langle I_{x}\right\rangle=K$ are physically meaningful as the vacuum states (not the vacuum band). The collective rotation in this case, therefore, is the rotation perpendicular to the symmetry axis which is at the same time the non-collective rotation axis ( $x$-axis), and the band consists of multiple wobbling phonons with excitation energy

$$
E_{\gamma}^{(\text {ver })}(K)=\hbar \omega_{w}(K)+\hbar \omega_{\text {rot }} \approx K / \mathcal{J}_{\perp}, \quad\left(\mathcal{J}_{\perp} \equiv \mathcal{J}_{y}=\mathcal{J}_{z}\right)
$$

where eqs.(3.5b) and 3.3) are used, and with the transfer angular momentum $\Delta I=+1$ excited on the high- $K$ band head (vacuum) states; namely,

$$
E_{|\Delta I|=1}^{(\text {ver })}(I) \approx\left(I(I+1)-K^{2}\right) / 2 \mathcal{J}_{\perp}+\text { const. }, \quad(I \gtrsim K)
$$


consistently to the large $I \approx K$ approximation. Note that the rotational frequency $\hbar \omega_{\text {rot }}=$ $K / \mathcal{J}_{x}$ disappears in the final expressions as it should be because it is not the collective rotational frequency in this case. This kind of rotational band is specifically called as a "precession-band" and often observed as a band excited on top of a high- $K$ isomer state

and has been well studied microscopically in both realistic nuclei ${ }^{26,27)}$ and in a schematic model. ${ }^{28)}$

From the microscopic view point, there is a collective motion even in the limit of axial symmetry of the "collective" rotation, $\gamma=0^{\circ}$ and $-60^{\circ}$ : that is nothing but the $\gamma$-vibration with signature $\alpha=1$ which has been just considered in the previous section. The reason why the limit does not well-behave is that the "body-fixed" frame or the "principal-axis" (see the next section) frame of the quadrupole tensor is not well defined in this limit. The calculation in the previous section corresponds to the one in the "uniformly-rotating" frame, which is always possible to define. It will be shown, explicitly in the next section, that the transformation to the "principal-axis" frame is impossible in the "collective" rotation limit from the microscopic view point.

\section{$\S 4$ Microscopic RPA Treatment}

Although the wobbling motion is a kind of oscillatory motion of the rotation axis, the shape degrees of freedoms are necessary to consider. Especially what is important is the non-diagonal parts of the quadrupole tensor and thus we introduce,

$$
\begin{aligned}
& Q_{y} \equiv Q_{1}^{(-)}=-\frac{1}{2} \sqrt{\frac{15}{4 \pi}} \sum_{a=1}^{A}(x z)_{a}, \\
& Q_{z} \equiv Q_{2}^{(-)}=i \frac{1}{2} \sqrt{\frac{15}{4 \pi}} \sum_{a=1}^{A}(x y)_{a},
\end{aligned}
$$

which just appear as the $\Delta I= \pm 1 E 2$ transition operators in eq.(2.3b). Note that only the modes with signature $\alpha=1$ are relevant in the RPA order so that only these modes are considered in the following. In higher order, however, one should also consider the remaining non-diagonal component, $Q_{x} \equiv Q_{1}^{(+)}$,, 5$)$ with all the RPA eigen modes.

In the RPA treatment, the in-band $\Delta I= \pm 2 E 2$ transition does not change the RPA phonon number and then the matrix elements in the vacuum (or yrast) band and the one-phonon wobbling band are the same. In contrast the interband transition from the 
one-phonon wobbling to the vacuum band reflects the nature of the wobbling motion itself. From the argument in $\S 2$ (see eqs.(2.1) and (2.3c)) we have

$$
B(E 2)_{\Delta I= \pm 2}^{(\text {in-band })} \approx\left\langle Q_{2 \pm 2}^{(E)}\right\rangle^{2}=\left(e \frac{Z}{A}\right)^{2} \frac{1}{2} R^{4}\left(\alpha_{y}-\alpha_{z}\right)^{2}
$$

where we have used eq.(3.8) with an understanding that the deformation parameters are those associated with the vacuum states, $\left|\omega_{\text {rot }}\right\rangle$, and

$$
B(E 2)_{n, \Delta I= \pm 1}^{(\text {inter })} \approx \frac{1}{2}\left(\left(\mathcal{Q}_{y}(n) \mp \mathcal{Q}_{z}(n)\right)^{(E)}\right)^{2}
$$

where $n$ means that the $n$-th RPA eigen mode is considered and the RPA amplitudes are defined as

$$
\mathcal{Q}_{k}(n) \equiv\left\langle n\left|Q_{k}\right| 0\right\rangle_{\mathrm{RPA}}=\left\langle\left[X_{n}, Q_{k}\right]\right\rangle \quad(k=y, z),
$$

with $X_{n}$ being the annihilation operator of the n-th RPA eigen mode. Note that $Q_{z}$ is anti-hermite while $Q_{y}$ is hermite and this is the reason why the sign in eq. $(4.2 \mathrm{~b})$ is changed from that in eq.(2.3b). Here and hereafter $\langle O\rangle$ for any operator $O$ means the expectation value with respect to the cranked state $\left|\omega_{\text {rot }}\right\rangle$. It is clear that the expression of the in-band E2 transition formally coincides with those of the horizontal transition in the macroscopic rotor model in eq.(3.7a). How about the interband transition? It is one of the main purpose of this section to clarify this point.

The part of operators, $Q_{y}$ and $Q_{z}$, relevant in the RPA order can be expanded in terms of the RPA eigen modes,

$$
\begin{aligned}
& Q_{y}=\sum_{n: \text { all }}\left(\mathcal{Q}_{y}(n) X_{n}^{\dagger}+\text { h.c. }\right), \\
& Q_{z}=\sum_{n: \text { all }}\left(\mathcal{Q}_{z}(n) X_{n}^{\dagger}-\text { h.c. }\right),
\end{aligned}
$$

Here $(n: a l l)$ means that the contribution of the Nambu-Goldstone (NG) mode,

$$
X_{\mathrm{NG}}^{\dagger}=\frac{1}{\sqrt{2 I}}\left(J_{z}+i J_{y}\right), \quad I \equiv\left\langle J_{x}\right\rangle
$$

should be included:

$$
\begin{aligned}
& \mathcal{Q}_{y}(n=\mathrm{NG})=-\frac{1}{\sqrt{2 I}} 2 R^{2} \alpha_{y}, \\
& \mathcal{Q}_{z}(n=\mathrm{NG})=\frac{1}{\sqrt{2 I}} 2 R^{2} \alpha_{z} .
\end{aligned}
$$


Namely the contribution of the NG mode corresponds to the "static" deformation while those of the normal mode to the "dynamic" (or vibrational) deformations. It should be mentioned that there exists a kind of "sum-rule" *) which relates both contributions:

$$
\sum_{n \neq \mathrm{NG}} \mathcal{Q}_{y}(n) \mathcal{Q}_{z}(n)=\frac{R^{4}}{I} 2 \alpha_{y} \alpha_{z}
$$

which can be easily verified from the identity,

$$
\left[Q_{y}, Q_{z}\right]=0
$$

Although it is not stated explicitly we are working in the so-called "uniformly rotating" (UR) frame in the sense that all observables are based on the vacuum state $\left|\omega_{\text {rot }}\right\rangle$ which is the cranked state uniformly rotating around the one of the principal axes of the deformation of the mean-field. The wobbling motion naturally appears in the body-fixed frame, or the "principal axis" (PA) frame." It is not a trivial matter to define the PA frame in the general framework of the many-body problem. One must introduce "gauge conditions" ${ }^{3,6)}$ which are common in the quantum theory with constraints. According to Refs., ${ }^{2,4)}$ we impose the non-diagonal part of the quadrupole tensor should vanish:

$$
\left(Q_{k}\right)_{\mathrm{PA}}=0 \quad(k=x, y, z) .
$$

The meaning of these condition are apparent. The PA and UR frame picture are related through the Euler angles which are now the dynamical variables, for example,

$$
\begin{gathered}
\left(J_{i}\right)_{\mathrm{UR}}=\sum_{k=x, y, z} D_{i k}(\Theta)\left(J_{k}\right)_{\mathrm{PA}}, \\
\left(Q_{i j}\right)_{\mathrm{UR}}=\sum_{k, l=x, y, z} D_{i k}(\Theta) D_{j l}(\Theta)\left(Q_{k l}\right)_{\mathrm{PA}},
\end{gathered}
$$

where $Q_{i j}$ is the non spherically-coupled representation of the quadrupole tensor, $Q_{k}=Q_{i j}$ (ijk-cyclic) and $D_{i j}(\Theta)$ is the rank-1 $D$ function with the additionally introduced Euler angles $\Theta$. The three conditions, eq.(4.9), in principle, define the microscopic structure of the three Euler angles in terms of the complete set of the observables in the UR frame,

*) Strictly speaking, it might not be called as a sum-rule because each term in the summation in eq.(4.7) is not positive definite. 
where every microscopic quantity is well-defined, and remove the redundancy between the microscopic variables in the PA frame and the collective variables $\Theta$. Of course it is not so simple: for instance, what is the ordering between the Euler angle operators and the microscopic quadrupole tensor in eq.(4.10b)? One must work out very carefully in a consistent framework.

Fortunately, it has been shown that the small amplitude approximation makes the situation quite simple. $^{2,6)}$ The Euler angles can be written in terms of the microscopic variables and then the transformation from the UR to PA frame can be done explicitly by using them within the RPA order, ${ }^{2)}$ for example,

$$
\begin{aligned}
\left(J_{x}\right)_{\mathrm{PA}} & =\left(J_{x}\right)_{\mathrm{UR}} \\
\left(i J_{y}\right)_{\mathrm{PA}} & =\left(i J_{y}-\frac{I}{2 R^{2} \alpha_{z}} Q_{z}\right)_{\mathrm{UR}} \\
\left(J_{z}\right)_{\mathrm{PA}} & =\left(J_{z}+\frac{I}{2 R^{2} \alpha_{y}} Q_{y}\right)_{\mathrm{UR}}
\end{aligned}
$$

Using the UR frame relations eq.(4.4), the angular momentum operators in the PA frame are written in terms of the microscopic RPA eigen modes,

$$
\begin{aligned}
\left(i J_{y}\right)_{\mathrm{PA}} & =-I \sum_{n \neq \mathrm{NG}}\left(\frac{\mathcal{Q}_{z}(n)}{2 R^{2} \alpha_{z}} X_{n}^{\dagger}-\text { h.c. }\right), \\
\left(J_{z}\right)_{\mathrm{PA}} & =I \sum_{n \neq \mathrm{NG}}\left(\frac{\mathcal{Q}_{y}(n)}{2 R^{2} \alpha_{y}} X_{n}^{\dagger}+\text { h.c. }\right)
\end{aligned}
$$

namely, the NG mode contribution disappears. Apparently the transformation from the UR to PA frame is possible only in the case when $\alpha_{y} \neq 0$ and $\alpha_{z} \neq 0$ (see eq.(4.21) below for more strict conditions). Now it is easy to check, with the help of the sum-rule relation, eq.(4.7), that (a part of) the commutation relation in the PA frame, the sign of which is opposite to the one in the UR frame, holds again within the RPA order, ${ }^{2)}$

$$
\left[\left(i J_{y}\right)_{\mathrm{PA}},\left(J_{z}\right)_{\mathrm{PA}}\right]=+I=\left\langle\left(J_{x}\right)_{\mathrm{PA}}\right\rangle .
$$

It should be noticed that the transformation from the laboratory frame to the UR frame is unitary while that from the UR to PA is not as is clear from this commutation relation. The physical reason why the transformation form the UR to PA is non-unitary is apparent: the Euler angles in eq.(4.10) are not simple parameters but are now considered to be the 
dynamical variables canonically corresponding to the collective angular momenta, which is a common feature to the theory with constraints. ${ }^{3,6)}$

Next, let us consider the dynamics in the PA frame. The time-dependence in the UR frame is governed by the hamiltonian,

$$
H_{\mathrm{UR}} \equiv H-\omega_{\mathrm{rot}} J_{x}
$$

Since the Euler angles are the dynamical variables describing the wobbling of the rotation axis, the hamiltonian in the PA frame are modified,

$$
\begin{aligned}
H_{\mathrm{PA}} & =H-\Omega_{x} J_{x}-\Omega_{y} J_{y}-\Omega_{z} J_{z} \\
& \approx H_{\mathrm{UR}}-\Omega_{y} J_{y}-\Omega_{z} J_{z}
\end{aligned}
$$

where the operators in the right hand side are in the UR frame (we omit () UR hereafter) $\Omega_{k} \quad(k=x, y, z)$ are angular frequency operators conjugate to the Euler angle operators and within the RPA order,

$$
\Omega_{x} \approx \omega_{\text {rot }} \gg \Omega_{y}, \Omega_{z}
$$

reflecting the small amplitude RPA ansatz. The microscopic structure of the angular frequency operators are determined by the consistency conditions of the gauge conditions, ${ }^{2,6)}$

$$
i \frac{d}{d t}\left(Q_{k}\right)_{\mathrm{PA}} \equiv\left(\left[Q_{k}, H_{\mathrm{PA}}\right]\right)_{\mathrm{PA}}=0 \quad(k=x, y, z) .
$$

Again within the RPA order, we obtain

$$
\begin{gathered}
i \Omega_{y}=-\frac{1}{2 \hbar R^{2} \alpha_{y}}\left(\left[H_{\mathrm{UR}}, Q_{y}\right]\right)_{\mathrm{PA}}=-\sum_{n \neq \mathrm{NG}}\left\{\left(\omega_{n} \frac{\mathcal{Q}_{y}(n)}{2 R^{2} \alpha_{y}}+\omega_{\mathrm{rot}} \frac{\mathcal{Q}_{z}(n)}{2 R^{2} \alpha_{z}}\right) X_{n}^{\dagger}-\text { h.c. }\right\}, \\
\Omega_{z}=\frac{1}{2 \hbar R^{2} \alpha_{z}}\left(\left[H_{\mathrm{UR}}, Q_{z}\right]\right)_{\mathrm{PA}}=\sum_{n \neq \mathrm{NG}}\left\{\left(\omega_{n} \frac{\mathcal{Q}_{z}(n)}{2 R^{2} \alpha_{z}}+\omega_{\operatorname{rot}} \frac{\mathcal{Q}_{y}(n)}{2 R^{2} \alpha_{y}}\right) X_{n}^{\dagger}+\text { h.c. }\right\},
\end{gathered}
$$

where the following results of the RPA eigen value problem in the UR frame are used in the second equality in each equation,

$$
H_{\mathrm{UR}} \approx \sum_{n: \text { all }} \hbar \omega_{n} X_{n}^{\dagger} X_{n}=\sum_{n \neq \mathrm{NG}} \hbar \omega_{n} X_{n}^{\dagger} X_{n}+\frac{\hbar \omega_{\mathrm{rot}}}{2 I}\left\{J_{z}^{2}-\left(i J_{y}\right)^{2}\right\} .
$$

Eqs.(4.12) and (4.18) show that both the angular momentum vector and the angular frequency vector precess around the main rotation axis (x-axis) in the PA frame with the amplitudes,

$$
\left(J_{k}\right)_{\mathrm{PA}}(n) \equiv\left\langle n\left|\left(J_{k}\right)_{\mathrm{PA}}\right| 0\right\rangle_{\mathrm{RPA}}, \quad \Omega_{k}(n) \equiv\left\langle n\left|\Omega_{k}\right| 0\right\rangle_{\mathrm{RPA}} \quad(k=y, z) .
$$


For this picture to be consistent the ratios of the dynamic to the static deformation should be small, i.e.,

$$
\begin{aligned}
& r_{y}(n) \equiv \mathcal{Q}_{y}(n) / 2 R^{2} \alpha_{y}=\left(J_{z}\right)_{\mathrm{PA}}(n) / I \sim O(1 / \sqrt{I}) \ll 1, \\
& r_{z}(n) \equiv \mathcal{Q}_{z}(n) / 2 R^{2} \alpha_{z}=-\left(i J_{y}\right)_{\mathrm{PA}}(n) / I \sim O(1 / \sqrt{I}) \ll 1,
\end{aligned}
$$

Now the "effective" moments of inertia ${ }^{2)}$ for each RPA eigen mode, which are naturally introduced through

$$
\hbar \mathcal{J}_{k}^{\text {eff }}(n) \equiv\left(J_{k}\right)_{\mathrm{PA}}(n) / \Omega_{k}(n) \quad(k=y, z),
$$

are thus written as,

$$
\begin{aligned}
\hbar \mathcal{J}_{y}^{\text {eff }}(n) & =\frac{I r_{z}(n)}{\omega_{n} r_{y}(n)+\omega_{\text {rot }} r_{z}(n)}, \\
\hbar \mathcal{J}_{z}^{\text {eff }}(n) & =\frac{I r_{y}(n)}{\omega_{n} r_{z}(n)+\omega_{\text {rot }} r_{y}(n)},
\end{aligned}
$$

where eqs.(4.12) and (4.18) and the definition (4.21) are used. Introducing the quantities (c.f. eq. (3.3c)),

$$
\begin{aligned}
& W_{y}(n) \equiv 1 / \mathcal{J}_{z}^{\text {eff }}(n)-1 / \mathcal{J}_{x}, \\
& W_{z}(n) \equiv 1 / \mathcal{J}_{y}^{\text {eff }}(n)-1 / \mathcal{J}_{x},
\end{aligned}
$$

we have

$$
\left\{\begin{array}{l}
\hbar \omega_{n}\left(r_{y}(n) / r_{z}(n)\right)=I W_{z}(n), \\
\hbar \omega_{n}\left(r_{z}(n) / r_{y}(n)\right)=I W_{y}(n),
\end{array}\right.
$$

from which the well-known wobbling energy formula is obtained as in the same way as in eq.(3.3a),

$$
\hbar \omega_{n}=I \sqrt{W_{y}(n) W_{z}(n)}=\hbar \omega_{\text {rot }} \sqrt{\frac{\left(\mathcal{J}_{x}-\mathcal{J}_{y}^{\text {eff }}(n)\right)\left(\mathcal{J}_{x}-\mathcal{J}_{z}^{\text {eff }}(n)\right)}{\mathcal{J}_{y}^{\text {eff }}(n) \mathcal{J}_{z}^{\text {eff }}(n)}} .
$$

Note that the right hand side of this equation depends on the eigen mode itself so that it is only a formal solution of the RPA equation, to which eq.(4.25) is equivalent. It may be worth noticing that eigen value eq.(4.25) can be obtained by the Euler equation for the PA angular momentum vector, which can be derived from the TDHF variational principle., In fact, the Euler equation with the PA hamiltonian eq.(4.15),

$$
\frac{d}{d t}\left(J_{i}\right)_{\mathrm{PA}}=\sum_{j k} \varepsilon_{i j k}\left(J_{j}\right)_{\mathrm{PA}} \Omega_{k}
$$


leads, for the component of the n-th RPA eigenmode,

$$
\left\{\begin{array}{l}
-\omega_{n}\left(i J_{y}\right)_{\mathrm{PA}}(n)=\Omega_{z}(n) I-\omega_{\mathrm{rot}}\left(J_{z}\right)_{\mathrm{PA}}(n) \\
-\omega_{n}\left(J_{z}\right)_{\mathrm{PA}}(n)=i \Omega_{y}(n) I-\omega_{\mathrm{rot}}\left(i J_{y}\right)_{\mathrm{PA}}(n)
\end{array}\right.
$$

which reduces to eq.(4.25) first by substituting $\Omega_{y, z}(n)=\left(J_{y, z}\right)_{\mathrm{PA}}(n) / \hbar \mathcal{J}_{k}^{\text {eff }}(n)$ (eq.(4.22)) and next by $\left(i J_{y}\right)_{\mathrm{PA}}(n)=-I r_{z}(n)$ and $\left(J_{z}\right)_{\mathrm{PA}}(n)=I r_{y}(n)$ (eq. (4.21)).

Since we are considering the case where eigen-energies of all RPA solutions are real and positive, the signs of $W_{y}(n)$ and $W_{z}(n)$ are the same and then the basic eq.(4.25) can be formally solved for $r_{y}(n), r_{z}(n)$,

$$
\begin{aligned}
& r_{y}(n)=c_{n} \frac{1}{\sqrt{2 I}}\left(\frac{W_{z}(n)}{W_{y}(n)}\right)^{\frac{1}{4}}, \\
& r_{z}(n)=\sigma_{n} c_{n} \frac{1}{\sqrt{2 I}}\left(\frac{W_{y}(n)}{W_{z}(n)}\right)^{\frac{1}{4}},
\end{aligned}
$$

where $\sigma_{n}$ denotes the sign of $W_{y}(n)$ (= the sign of $\left.W_{z}(n)\right)$ and the quantity $c_{n}$ is the amplitude with which the n-th mode contributes to the sum-rule (4.7),

$$
\sum_{n \neq \mathrm{NG}} r_{y}(n) r_{z}(n)=\frac{1}{2 I} \Longleftrightarrow \sum_{n \neq \mathrm{NG}} \sigma_{n} c_{n}^{2}=1
$$

so that $c_{n}^{2} \ll 1$ for non-collective solutions and $c_{n}^{2} \approx 1$ for collective solutions. Using the definition of $r_{y}(n)$ and $r_{z}(n)$ (eq.(4.21)), eq.(4.29) shows that the transition amplitudes $\mathcal{Q}_{y}(n)$ and $\mathcal{Q}_{z}(n)$ can be expressed in terms of the static deformation parameters and the effective moments of inertia. Inserting them into eq.(4.2b), thus, we finally obtain,

$$
B(E 2)_{n, \Delta I= \pm 1}^{\text {(inter) }} \approx\left(e \frac{Z}{A}\right)^{2} \frac{1}{I} R^{4} c_{n}^{2}\left(\alpha_{y}\left(\frac{W_{z}(n)}{W_{y}(n)}\right)^{\frac{1}{4}} \mp \sigma_{n} \alpha_{z}\left(\frac{W_{y}(n)}{W_{z}(n)}\right)^{\frac{1}{4}}\right)^{2}
$$

where we assumed

$$
\mathcal{Q}_{k}^{(E)}(n) \approx e \frac{Z}{A} \mathcal{Q}_{k}(n) \quad(k=y, z)
$$

which are approximately satisfied for the (isoscalar) collective RPA solutions. Now the analogy with the eq. $(3.7 \mathrm{~b})$ (with $n_{w}=1$ ) in the macroscopic rotor model is clear: the main difference, except to the assumption (4.32), is the fact that there are many solutions in the microscopic RPA treatment and the amplitude $c_{n}^{2} \neq 1$ and the sign $\sigma_{n}$ can be negative. Note that the negative $\operatorname{sign} \sigma_{n}=-1$ means $\mathcal{J}_{y, z}^{\text {eff }}(n)>\mathcal{J}_{x}$ or $\mathcal{J}_{y, z}^{\text {eff }}(n)<0$, which 
conflicts our basic assumptions that the $\mathrm{x}$-axis is the main rotation axis and the introduced three moments of inertia are physically meaningful, and therefore clearly shows that the interpretation of the n-th RPA mode as a wobbling motion is not justified in such a case. From this observation we use positiveness of the sign $\sigma_{n}$, i.e. $r_{y}(n) r_{z}(n)>0$, and $c_{n}^{2} \approx 1$ as criteria to identify the wobbling solutions among the microscopic RPA eigen modes in later applications.

For completeness, the microscopic expression for the $M 1$ rate corresponding to the $\Delta I= \pm 1 E 2$ transitions, eq.(4.2b), is given:

$$
B(M 1)_{n, \Delta I= \pm 1}^{\text {inter })} \approx \frac{1}{2}\left(i \mu_{y}(n) \pm \mu_{z}(n)\right)^{2}
$$

with

$$
\mu_{k}(n) \equiv\left\langle n\left|\mu_{k}\right| 0\right\rangle_{\mathrm{RPA}}=\left\langle\left[X_{n}, \sqrt{\frac{3}{4 \pi}} \mu_{N}\left(g_{l} l_{k}+g_{s} s_{k}\right)\right]\right\rangle \quad(k=y, z),
$$

where $g_{l}$ and $g_{s}$ are usual orbital and spin g-factors. We here are mainly concerned with the even-even nuclei so that only the vibrational contribution are included. ${ }^{17)}$ In contrast to the case of $E 2$ transitions, where is used the same basic requirement as in the rotor model such that the quadrupole tensor is diagonal in the PA frame, there is no simple correspondence in M1 transitions between the results of the microscopic RPA framework and the macroscopic model. This is because the basic assumption of the $M 1$ operator in the rotor model, eq.(3.11), is not always justified from the microscopic viewpoint: for instance the effects of quasineutron or quasiproton alignments on the $M 1$ transitions cannot be taken into account. If we nevertheless take the same assumption as the rotor model, the operators responsible to the $M 1$ transitions are ${ }^{*)} i \mu_{y} \propto\left(g_{y}-g_{x}\right)\left(i J_{y}\right)_{\mathrm{PA}}, \mu_{z} \propto\left(g_{z}-g_{x}\right)\left(J_{z}\right)_{\mathrm{PA}}$. Then using eqs.(4.11), (4.21) and (4.29) a similar expression to the one in the rotor model, eq.(3.13), is easily obtained with the same notice as in the $E 2$ transitions, eq.(4.31).

In the following let us discuss general features of the high-spin RPA eigen modes. The properties of the solutions can be different from case to case depending on the microscopic structure of vacuum states. Until up to now, we have used the term "collective" somewhat ambiguously. It might be necessary to make it more precise here. The collectivity is

*) Note that only the component of the magnetic moment vector perpendicular to the angular momentum in the PA frame, $\boldsymbol{m}-g_{x} \boldsymbol{I}$, contributes to the transition. 
usually referred, at least, in two different contexts: (1) the transition matrix elements of characteristic observables to the solutions, e.g. the $E 2$ transition for the $\beta, \gamma$-vibrations and the wobbling motion under discussions, are large, and (2) the RPA amplitudes of phonon operators spread over many two-quasiparticle states. For example, it is the second one that is necessary for eq.(4.32) to be valid. Let us call the former the "large-transition" collectivity and the latter the "spread-over" collectivity. The shape vibrational motions in the ground state regions almost always satisfy the conditions of both collectivities. From our experiences, the spread-over collectivity gets weaker in most cases for the RPA solutions excited on the vacuum with rotational aligned quasiparticles, e.g. the s-band, at higher spins. One of the reasons for this is that the pairing correlations are reduced by the blocking effects of the aligned particles. ${ }^{29)}$ In spite of the fact that the phonon amplitudes somewhat tend to concentrate on few components, the large-transition collectivity can survive even at higher-spins, since the energy lowering of the two-quasiparticle states make the RPA eigen energy small so that the backward amplitudes remain to be large.

The discussions above indicates that the $M 1$ amplitude with using the microscopic M1 operator, eq.(4.34), is small for the typical spread-over collective RPA solutions. In fact a destructive interference occurs for the $M 1$ operator, because amplitudes of this type of solutions, which are of isoscalar quadrupole character, have many two-quasiparticle components with phases favourable to the E2 operator. Actually the quadrupole shape vibrations in non-rotating nuclei have RPA amplitudes which transfer the $K$ quantum number by even unit so that the transition matrix elements for the $M 1$ operator strictly vanish. This is well established for the $\gamma$-vibrational bands at low spins, where the $M 1$ transitions are usually small. Therefore the $\Delta I= \pm 1$ transitions are $E 2$ dominant in such cases. It should, however, be noticed that the spread-over collectivity of the RPA solutions are reduced at higher-spins and then the properties of individual two-quasiparticle states, whose components are dominant in the RPA amplitudes, manifest themselves as an enhancement of the $M 1$ amplitudes. We will see examples of such cases, where the $M 1$ transitions are non-negligible or even dominant, in the next section. 


\section{$\S 5$ Examples of Numerical Calculations}

In the microscopic RPA formalism we have solutions of vibrational character in many cases. As it is clarified in the previous sections, however, all of the solutions cannot necessarily be interpreted as the wobbling-like motions even though they are collective enough in the sense that the E2 transition amplitudes are large (see discussions below eq.(4.31)). Namely, in addition to the enough collectivity, the relative sign of the transition amplitudes $\mathcal{Q}_{y}(n)$ and $\mathcal{Q}_{z}(n)$ should be the same as that of the static asymmetries around $y$ and $z$-axes, $\alpha_{y}$ and $\alpha_{z}$,

$$
\operatorname{sign} \text { of }\left(\mathcal{Q}_{y}(n) / \mathcal{Q}_{z}(n)\right)=\operatorname{sign} \text { of }\left(\alpha_{y} / \alpha_{z}\right)
$$

This relation was mentioned in another form in eq.(4.9) of Ref.. ${ }^{25)}$ Only in such solutions the three moments of inertia associated with the RPA solutions, eq.(4.22), are well defined and are consistent to the picture of the wobbling motion. Interestingly enough, the lowest collective RPA solutions, if they exist, satisfy the condition in most of the cases we found. It might be interesting to note that eq.(5.1) holds also for $\gamma$-vibrational excitations in nuclei with small selfconsistent $\gamma$ deformation: It is a good approximation to set $\gamma=0$ in such nuclei and then the same relative sign as the selfconsistent triaxiality (eq.(5.1)) is obtained for the transition amplitudes even with such an artificial setting of $\gamma=0$.

More precisely speaking, one must also require the conditions eq.(4.21) in order for the adopted small amplitude approximation to be valid. This means that both static deformation around $y$ and $z$-axes should be larger than the dynamic ones. Therefore, one might expect the ideal wobbling motion only in the region of equilibrium shape with $\gamma \neq 0^{\circ}$ and $\gamma \neq-60^{\circ}$ in the $\left(\epsilon_{2}, \gamma\right)$ plane; i.e. an appreciable amount of triaxiality is generally required. Notice that the wobbling-like solutions can exist in the axially symmetric cases with $\gamma=60^{\circ}$ and $=-120^{\circ}$, where $\alpha_{y}=\alpha_{z}<0$ and $>0$, respectively, and correspond to the $\Delta I=1$ excitations on a high- $K$ isomer states (the precession-bands) as is discussed in the end of $\S 3$.

This apparent asymmetry between the four cases of axially symmetric shapes are based on the fact that the three rotation axes are not treated equivalently: the $x$-axis is the main rotation axis and the angular momenta around the other two axes are small compared to it, which is generally believed to be valid near the yrast states. Such a treatment 
especially makes sense in the situation where the quasiparticle alignments occur: then an appreciable part of the main rotation are carried by single-particle degrees of freedoms, while perpendicular components of the rotation are only of collective nature. Actually, we have found the wobbling-like solutions only in the cases where aligned quasiparticles exist in the vacuum state from which the RPA modes are excited, which makes the vacuum time-reversal broken and induces strong rotational $K$-mixing effects.

With the general considerations above in mind we will show some examples of the results of realistic calculations and discuss the characteristic properties of the obtained wobbling motions.

\section{$\S 5-1 \Delta I= \pm 1$ interband E2 transitions}

Restricting ourselves to even-even nuclei, the yrast band has naturally the quantum numbers, $(\alpha, \pi)=(0,+)$ with even-spin values. Then the (one-phonon) wobbling band has $(\alpha, \pi)=(1,+)$ with odd-spin values. Unfortunately, there are only few cases where $(\alpha, \pi)=(1,+)$ bands are observed up to high-spin states. Moreover it is generally difficult to distinguish the collective phonon band from non-collective quasiparticle bands, like the $A C$ or $B D$ two-neutron aligned bands in the usual nomenclature of quasiparticle orbits, only from the energy spectra. Therefore, at least until now, there is no definite evidence that the ideal wobbling motions exist in atomic nuclei.

One of the most important feature of the wobbling motions is that the E2 amplitudes $\mathcal{Q}_{y}(n)$ and $\mathcal{Q}_{z}(n)$ have comparative magnitude, in contrast to the case of the $\gamma$-vibrations in $\S 2$, where $\left|\mathcal{Q}_{y}\left(=t\left[Q_{1}^{(-)}\right]\right)\right| \ll\left|\mathcal{Q}_{z}\left(=-t\left[Q_{2}^{(-)}\right]\right)\right|$. This property immediately leads that the one of the transitions with either $\Delta I=+1$ or $=-1$ is much larger than the other depending on the relative phase of $\mathcal{Q}_{y}(n)$ and $\mathcal{Q}_{z}(n)$, see eq.(4.2b), or the triaxiality, see eq.(5.1). This is situation quite analogous to the $M 1$ transitions between the signature partner bands in odd nuclei, where the single-particle matrix elements of the operators $i \mu_{y}$ and $\mu_{z}$ between the signature partner bands play a similar role to the two amplitudes $\mathcal{Q}_{y}$ and $\mathcal{Q}_{z}$, see Figure 2, although the role played by the triaxiality is not necessarily the same. For the ideal case of the wobbling motion where the formula of the macroscopic model are valid, the staggering of the $E 2$ transitions between the $\Delta I=+1$ and -1 combined with the excitation energy gives important information on the triaxial deformation and/or the 
three moments of inertia; for example,

$$
\frac{\Delta B(E 2)_{|\Delta I|=1}}{B(E 2)_{|\Delta I|=2}} \equiv \frac{B(E 2)_{\Delta I=-1}-B(E 2)_{\Delta I=+1}}{B(E 2)_{|\Delta I|=2}}=\frac{8}{I} \frac{\alpha_{y} \alpha_{z}}{\left(\alpha_{y}-\alpha_{z}\right)^{2}},
$$

just as in a similar kind of analysis for the staggering of the $M 1$ transitions in odd nuclei as in Ref.. ${ }^{30)}$ The relation between the $B(E 2)_{\Delta I= \pm 1}$ and the triaxial equilibrium deformation is summarized in Figure 3.

Although there is no definite evidence, it is suggested in Ref. ${ }^{25)}$ that the odd-spin sequence of the so-called "extention of the $\gamma$-band" ${ }^{31)}$ after the $g$ - $s$ band crossing in ${ }^{182}$ Os might be a candidate of the wobbling motion from the calculations of the same RPA formalism. It is instructive here to show the results for this nucleus because the results of the calculations show an ideal feature discussed in the previous sections. This nucleus is supposed to be $\gamma$-soft and the two quasineutron alignment induce the negative $\gamma$ deformation. Although the potential energy surface are rather flat so that it is difficult to determine the precise $\gamma$ value, the RPA results with $\gamma=-18^{\circ}$ has been shown to have desired property as an ideal wobbling motion. ${ }^{12,25)}$ It should be stressed that in order to obtain the wobbling-like RPA solution the existence of the aligned quasiparticles in the vacuum configuration, e.g. the $s$-band, is indispensable.

The calculational procedures, the single particle potential, the effective interactions and the model space etc, are the same as those in $\S 2$. It should, however, be stressed that the RPA dispersion equation with the NG modes being explicitly decoupled ${ }^{16)}$ is used in this calculations. For the case with $\gamma \neq 0^{\circ}$, then, the quadrupole force parameters disappears for the signature $\alpha=1$ sector. Therefore the wobbling-like RPA solution are obtained without any ambiguity depending on the choice of the force parameters. Just as in Ref., ${ }^{25)}$ all the mean-field parameters are fixed, for simplicity, with the values

*) In Refs., ${ }^{125)}$ the transition amplitudes are 10-30\% larger because a larger model space $\left(N_{\text {osc }}=5-7\right.$ for neutrons and $=4-6$ for protons $)$ than that adopted in this paper has been used. From the results in $\S 2$ we hope the calculations in this paper are more realistic. In addition, the results in Ref. ${ }^{25)}$ were obtained by using the RPA equation without the NG-modes explicitly decoupled. The rotational frequency dependence of the excitation energy of the wobbling is thus a little bit different form that in Ref., ${ }^{12)}$ where the same NG-decoupled equation as the present paper was used. 
$\Delta_{\nu}=0.74, \Delta_{\pi}=0.98 \mathrm{MeV}$ and $\epsilon_{2}=0.21, \gamma=-18^{\circ}$, which are roughly appropriate for the $s$-band of ${ }^{182}$ Os. The results of the RPA amplitudes for the lowest RPA solution and the corresponding $B(E 2)$ values are shown as functions of the rotational frequency in Figure 4. Since $-60^{\circ}<\gamma<0^{\circ}, \alpha_{y}<0$ and $\alpha_{z}>0$, and then the relative sign of $\mathcal{Q}_{y}(n)$ and $\mathcal{Q}_{z}(n)$ for the solution is negative (see eq.(5.1)). Thus, the transitions from $(I-1)_{\text {wob }}$ to $(I)_{s}$ is much stronger than the one from $(I+1)_{\text {wob }}$ to $(I)_{s}$, as is clear in Fig.4 (see also Fig.3), so that the $B(E 2)$ values show remarkable zigzag behaviour.

Unfortunately these transition rates have not yet been measured. Since the $\gamma$-ray energy for the $(I-1)_{\text {wob }} \rightarrow(I)_{s}$ transition is much smaller, the $\gamma$ decay rate for this transition, which has larger $B(E 2)$, is hindered. This might be the reason why it is difficult to measure the transitions. As clear from the general consideration, if the wobbling type RPA solution exists for the positive $\gamma$ equilibrium shape, the relative sign of $\mathcal{Q}_{y}(n)$ and $\mathcal{Q}_{z}(n)$ is positive and then both the $B(E 2)$ and the $\gamma$-ray energy for the $(I+1)_{\text {wob }} \rightarrow(I)_{s}$ transition is larger. Therefore, it might be more easy to measure the transitions in this case, although we could not find a good example of such calculations.

The $M 1$ transitions between the wobbling and the $s$-band in this nucleus is small, typically $B(M 1)\left(\mu_{N}^{2}\right) / B(E 2)\left(\mathrm{e}^{2} \mathrm{~b}^{2}\right) \approx 0.1$, except the highest frequency shown in Fig.4, where $M 1$ amplitudes start to grow rapidly. The smallness of the $M 1$ amplitudes comes from the fact that the wobbling solution in this nucleus is an ideal case and keep large collectivity up to rather high-spins (see the discussion at the end of $\S 4$ ), although it reduces gradually as is seen from the $B(E 2)$-values in Fig.4.

Finally, it should be mentioned that the second RPA solution in this nucleus is also considerably collective. ${ }^{25)}$ However, the relative sign of $\mathcal{Q}_{y}(n)$ and $\mathcal{Q}_{z}(n)$ is positive and therefore it is a kind of vibrational mode but is not of wobbling nature.

\section{§5-2 Effective moments of inertia}

Another important outcome of the microscopic RPA formalism is that the three effective moments of inertia ${ }^{2)}$ can be calculated, which are highly nontrivial from the microscopic viewpoint. It should be noticed that the ratios $\mathcal{J}_{y}^{\text {eff }}(n) / \mathcal{J}_{x}$ and $\mathcal{J}_{z}^{\text {eff }}(n) / \mathcal{J}_{x}$ can be extracted from the ratios $\omega_{n} / \omega_{\text {rot }}$ and $\mathcal{Q}_{y}(n) / \mathcal{Q}_{z}(n)$ through eqs.(4.26) and (4.29); the latter ratios are experimentally observable from the energy spectra and the 
$B(E 2)_{|\Delta I|=1} / B(E 2)_{|\Delta I|=2}$. It may therefore be interesting to see how the three moments of inertia calculated from the wobbling RPA solution behaves as functions of deformation parameters, especially the triaxiality $\gamma$. Their dependence on the rotational frequency have been already studied in Ref. ${ }^{25)}$ for ${ }^{182}$ Os, and will be presented for ${ }^{124} \mathrm{Xe}$ in the next subsection. In the case of the quadrupole residual interaction, a simple formula for $\mathcal{J}_{y, z}^{\text {eff }}(n)$ in terms of the $2 \times 2$-coupled RPA dispersion equations exists. ${ }^{16,25)}$ We have used this formula in the following calculations in place of the original definition, eq.(4.22).

Of course the true equilibrium shape has a definite $\gamma$ deformation. Here, however, we fixed the other parameters of the mean-field potential and have performed the RPA calculations with changing the $\gamma$ deformation as a free parameter. Thus, it should be considered to be a kind of theoretical simulations how the nucleus behaves if the triaxiality is artificially changed. The results for the three sectors in the $\left(\epsilon_{2}, \gamma\right)$ plane, $-120^{\circ}<\gamma<$ $-60^{\circ},-60^{\circ}<\gamma<0^{\circ}$ and $0^{\circ}<\gamma<60^{\circ}$, in each of which a representative nucleus, ${ }^{176} \mathrm{Hf},{ }^{182} \mathrm{Os}$ and ${ }^{148} \mathrm{Gd}$, is respectively selected, are shown together in one panel in Figure 5. Note that the yrast sequences of ${ }^{176} \mathrm{Hf}$ and ${ }^{148} \mathrm{Gd}$ are known to be composed of noncollective rotations with prolate and oblate shapes, i.e. $\gamma=-120^{\circ}$ and $=60^{\circ}$, respectively. Since all the other mean-field parameters are fixed with neglecting the selfconsistency, the wobbling-like solutions are not obtained for all values of $\gamma$ deformation, and, moreover, the solutions can be discontinuous as functions of $\gamma$ because of the existence of virtual level crossings of the quasiparticle orbits when changing $\gamma$.

In spite of the deficiencies of this relatively simple-minded calculations, it is instructive to see the microscopically derived $\gamma$ dependence of the three moments of inertia, which is neither of irrotational like nor of rigid-body like. In these calculations all the vacuum states, on which the RPA mode excited, have aligned quasiparticles, which is essential to obtain the wobbling-like solutions as is mentioned above, so that the $\mathcal{J}_{x}=I / \hbar \omega_{\text {rot }}$ (kinematical moment of inertia) changes very gradually and never vanish. Thus the $\gamma$ dependence of the three moments cannot be that of irrotaional. On the other hand $\mathcal{J}_{k}^{\text {eff }} \quad(k=y, z)$ are dynamical moments of inertia and take as small values as zero. Thus the $\gamma$ dependence of the three moments cannot be that of rigid-body, either.

It is very interesting to see how the three moments of inertia, which can be experi- 
mentally extracted from the observed wobbling motions, if they exist, behave. For this purpose, the triaxial deformation should be also determined from the independent experimental observables, e.g. by the $M 1$ transitions in neighbouring nuclei.

\section{§5-3 Wobbling motion in ${ }^{124} \mathrm{Xe}$}

As pointed out above, one of possible candidates of wobbling motion might be the highspin continuation of the odd-spin members of the so-called $\gamma$-band. Actually a scenario of character change from the $\gamma$-vibrational to the wobbling-like band was first pointed out in Ref., and such a trend, though not well developed because of the small triaxiality, are suggested for ${ }^{164} \mathrm{Er}$ in Ref.. ${ }^{16)}$ The essential point is that a structural change of the vacuum caused by the quasiparticle alignment transfers an appreciable amount of $K=1$ quadrupole strength from the Nambu-Goldstone mode to the normal modes. Although it is difficult to predict precisely in which nuclei wobbling mode appears because this mode is an outcome of subtle interplay between rotation, quadrupole and pairing correlations in realistic nuclei, we have at least up to now two typical candidates of wobbling motion in observed bands with negative $\gamma$ deformation: One is in ${ }^{182}$ Os after the $\left(\nu i_{13 / 2}\right)^{2}$ alignment discussed in Ref. ${ }^{25)}$ and above, and the other is in ${ }^{124} \mathrm{Xe}^{32)}$ after the $\left(\nu h_{11 / 2}\right)^{2}$ alignment being discussed in the following. ${ }^{*}$ An important characteristic common to both nuclei is that the low-spin spectrum is almost rotational but relatively $\gamma$-soft while negative $\gamma$ deformation, $-30^{\circ}<\gamma<0^{\circ}$ in ${ }^{182} \mathrm{Os}$ and $-60^{\circ}<\gamma<-30^{\circ}$ in ${ }^{124} \mathrm{Xe}$, is stabilized above the first band crossing caused by the high- $\Omega$ quasiparticles. Here we note that in ${ }^{182} \mathrm{Os}$ the $\gamma$-ray which links (the candidate of) the odd-spin members of the $\gamma$ band above and below the band crossing has not been observed. From this fact, we imagine it might well be the case that there might exist wobbling-like bands but have been assigned incorrectly as non-collective two-quasiparticle bands in other nuclei because of the lack of the linking transitions. A possible mechanism of this lack will be discussed later in $\S 5-4$ for the case of ${ }^{126} \mathrm{Ba}$.

The low-spin nuclear structure of Xe isotopes has been studied extensively by means of the interacting boson model ${ }^{33)}$ and known to depend smoothly on the neutron number. A recent study, ${ }^{34)}$ however, pointed out that above the first band crossing there are some

*) We thank R. Wyss for informing us of these data. 
properties which show sudden changes between $A=120$ and 122 due to the shape coexistence; the lighter isotopes remain nearly axially symmetric while the heavier ones become triaxial with $\gamma<0$. In particular, in ${ }^{120} \mathrm{Xe}$, a candidate of the even-spin continuation of the $\gamma$ band above the second band crossing has been found for the first time, whereas in ${ }^{124} \mathrm{Xe}$, the odd-spin sequence has been known to extend up to higher-spins than the even-spin one ${ }^{32)}$ and it shows well-developed wobbling character. We mainly concentrate on ${ }^{124} \mathrm{Xe}$ in the following, although similar results can be obtained for other isotopes with similar triaxial deformation.

The result of the RPA calculation for ${ }^{124} \mathrm{Xe}$ based on the $\left(\nu h_{11 / 2}\right)^{2}$ aligned configuration is shown in Figure 6 together with the experimental data seen from a reference which makes the routhian of the $s$-band flat. The method of the calculation are the same as in the previous sections but the smaller model space, $N_{\mathrm{osc}}=3-5$ for both neutrons and protons are used in this region of nuclei. This calculation have been done with using the deformation parameters $\epsilon_{2}=0.19$ and $\gamma=-45^{\circ}$, which are taken from the Total Routhian Surface (TRS) calculation at $\hbar \omega_{\text {rot }}=0.3 \mathrm{MeV}$ in Ref.. ${ }^{35)}$ The hexadecapole deformation is neglected for simplicity. Although the meaning of the shape parameters is slightly different because of the different single-particle potential adopted in Ref., ${ }^{35)}$ we confirmed the stability of results of our calculations against possible small change of shape parameters. The pairing gaps $\Delta_{\pi}=1.1 \mathrm{MeV}$ and $\Delta_{\nu}=0.9 \mathrm{MeV}$ typical for the low spin part of the neutron $s$-bands in this region are used. This RPA calculation reproduces the data very well. Note, again, that we do not have any adjustable parameter in the step of RPA for the signature $\alpha=1$ sector. Calculated effective moments of inertia are shown in Figure 7. They vary as functions of the rotational frequency in spite of the fact that the nuclear shape is fixed as in the case of ${ }^{182} \mathrm{Os}^{25)}$ but the dependence is much weaker in ${ }^{124} \mathrm{Xe}$.

Calculated $E 2$ and $M 1$ transition amplitudes are shown in Figures 8 and 9, respectively, as functions of the rotational frequency, where the standard values of $g_{l}$ and $g_{s}^{(\text {free) 36) }}$ and $g_{s}^{\text {(eff) }} / g_{s}^{\text {(free) }}=0.7$ are used in this and the following calculations for the $M 1$ operators, eq.(4.34). In the figures, the transition probabilities are also included. Since the selfconsistent $\gamma=-45^{\circ}$, the relative phase between $\mathcal{Q}_{y}$ and $\mathcal{Q}_{z}$ is negative so that the 
$\Delta I=+1$ transitions are stronger as in the same way as in ${ }^{182}$ Os. It is, however, noticed that the collectivity of the RPA solution, especially the spread-over one, is weaker than that in ${ }^{182} \mathrm{Os}$, and consequently the $M 1$ transitions is stronger in ${ }^{124} \mathrm{Xe}$. Actually, as is seen from Figs.8 and 9, $M 1$ transitions can compete with $E 2$ depending on the transition energies. The zigzag behaviour of the $E 2$ and $M 1$ transitions are the same in this case, see Figs.8 and 9. It might be interesting to point out that trends that the $E 2$ transitions decrease against the spin while the $M 1$ transitions increase coincide with the prediction of the macroscopic rotor model in this case, see eqs.(3.7b) and (3.13).

We use the quadrupole residual interaction which has two components, $K=1$ and $K=2$, in the signature $\alpha=1$ sector. Note that a triaxial deformation mixes $K$ quantum number by two unit so that the $K=1$ and 2 components of the interaction couple only through the rotational motion. It is the interplay of these two components intermediated by the rotational coupling that brings about the character change of the lowest collective excitation. To look at this mechanism more closely, it is useful to see the second-lowest RPA solution together with the lowest one that we have been discussing. The excitation energies of the two solutions and the $K=1$ and 2 quadrupole transition amplitudes, $\mathcal{Q}_{y}\left(=t\left[Q_{1}^{(-)}\right]\right)$and $\mathcal{Q}_{z}\left(=-t\left[Q_{2}^{(-)}\right]\right)$, associated with them, calculated at $\hbar \omega_{\text {rot }}=0.3 \mathrm{MeV}$ are shown in Figure 10 as functions of $\gamma$ from $-60^{\circ}$ to $0^{\circ}$. The calculation have been done using the same mean-field parameters (except $\gamma$ ) as in Fig.6. The second-lowest solution shares an appreciable amount of $\mathcal{Q}_{y}(n)$ and $\mathcal{Q}_{z}(n)$ strength with the lowest one but with the different relative sign between them. The similar situation has already been encountered in the case of ${ }^{182} \mathrm{Os}^{25)}$ In order to interpret these results, here we consider the axially symmetric limits, $\gamma=0^{\circ}$ and $-60^{\circ}$, where the symmetry axes are $z$ and $y$ axis, respectively. Since the role of $y$ and $z$ axes are interchanged (with the rotation axis unchanged) in both cases, the modes with $\left|\mathcal{Q}_{z}\right| \gg(\ll)\left|\mathcal{Q}_{y}\right|$ in the $\gamma=0^{\circ}$ on one hand corresponds to those with $\left|\mathcal{Q}_{y}\right| \gg(\ll)\left|\mathcal{Q}_{z}\right|$ in $\gamma=-60^{\circ}$ on the other hand. We can see clearly in Fig.10 that this correspondence is actually holds and, moreover, that the strongest $K$-mixing of the two amplitudes occurs at around $\gamma=-30^{\circ}$. If we call the mode with $\left|\mathcal{Q}_{z}\right| \gg\left|\mathcal{Q}_{y}\right|$ in the $\gamma=0^{\circ} " \gamma$-vibration like", then so is the second-lowest solution in ${ }^{124} \mathrm{Xe}$ at the rotational frequency shown. In contrast the lowest solution is " $\gamma$-vibration 
like" in ${ }^{182}$ Os at $\hbar \omega_{\text {rot }} \lesssim 0.25 \mathrm{MeV}$ as is shown in Fig.4. It should, however, be noticed that the lowest solution follows the "phase rule", eq.(5.1), which allows us to interpret the lowest solution as a wobbling motion in both cases. According to the previous discussions the $\Delta I=+1 E 2$ transitions are larger for the lowest solution because $\left(\mathcal{Q}_{y} / \mathcal{Q}_{z}\right)<0$, while the $\Delta I=-1 E 2$ transitions are larger for the second-lowest solution. No candidates of the second-lowest collective excitation has been observed so far. According to the TRS calculation, $^{35)}$ the equilibrium shape of the $\left(\nu h_{11 / 2}\right)^{2}$ band of ${ }^{122} \mathrm{Xe}$ is $\gamma \simeq-30^{\circ}$, where the excitation energy of the second-lowest solution becomes low and the difference between $B(E 2)_{\Delta I= \pm 1}$ becomes conspicuous due to the $K$-mixing. So we think ${ }^{122} \mathrm{Xe}$ is a more promising nucleus for which our theoretical prediction can be tested.

\section{§-4 Continuation of $\gamma$-band in ${ }^{126} \mathrm{Ba}$}

Next we study the high-spin continuation of the $\gamma$ band in ${ }^{126} \mathrm{Ba}^{37)}$ This nucleus is also relatively $\gamma$-soft at low spins, while the first band crossing is caused by the low- $\Omega\left(\pi h_{11 / 2}\right)^{2}$ in contrast to the isotone ${ }^{124}$ Xe studied above. See Fig.6 of Ref. ${ }^{35)}$ for a summary of the data. The alignment of high- $j$, low- $\Omega$ quasiparticles drives the nucleus towards $\gamma \gtrsim 0$. We have performed the RPA calculation for excitations on top of the $\left(\pi h_{11 / 2}\right)^{2}$ configuration adopting $\epsilon_{2}=0.24$ and $\gamma=0$ according to the TRS calculation ${ }^{37)}$ just as in the case of ${ }^{124} \mathrm{Xe}$. On the other hand the pairing strengths are chosen so as to reproduce their band crossing frequencies (Fig.12 of Ref. $\left.{ }^{37)}\right)$ both for proton $\left(\hbar \omega_{\mathrm{c}} \simeq 0.35 \mathrm{MeV}\right)$ and neutron $(\simeq$ $0.44 \mathrm{MeV}$ ) when the gap selfconsistent calculation has been done. We also confirmed that the parameters thus adopted reproduce the observed $B\left(E 2: 2_{1}^{+} \rightarrow 0_{1}^{+}\right)=1.9 \pm 0.2 \mathrm{e}^{2} \mathrm{~b}^{2}$ at the ground state. The calculated pairing gaps for the proton $s$-band are $1.16 \geq \Delta_{\nu} \geq 1.03$ $\mathrm{MeV}$ and $0.76 \geq \Delta_{\pi} \geq 0.71 \mathrm{MeV}$ for $0.4 \leq \hbar \omega_{\text {rot }} \leq 0.5 \mathrm{MeV}$. Since $\gamma \approx 0^{\circ}$ in this case we cannot use the RPA equation with the NG-mode fully decoupled. We therefore have used the equation with the NG-mode partially decoupled ${ }^{16)}$ suitable for $\gamma=0^{\circ}$, where the force parameter $\chi_{2}^{(-)}$remains and has been fixed so as to give the correct excitation energy of the $\gamma$ vibration on the ground state as $\hbar \omega_{\gamma}\left(\hbar \omega_{\text {rot }}=0\right)=0.873 \mathrm{MeV}$.

The result of the calculated routhian is shown in Figure 11 together with the data seen from a reference which makes the routhian of the $s$-band flat, while the $E 2$ and $M 1$ amplitudes are depicted in Figures 12 and 13 with corresponding transition probabilities 
included. The excitation energy decreases as the rotational frequency increases both in the data and calculation; this is contrary to the macroscopic wobbling motion. This result, together with the fact that the calculated signature-splitting of energy between $\alpha=0$ (not shown) and = 1 RPA solutions is very small, favours an interpretation that the band under consideration remains to be $\gamma$-vibration like rather than evolving to wobbling like above the band crossing, as in the case of ${ }^{164} \mathrm{Er}^{16)}$ In fact, the mixing of the $K=1$ component is not so strong, as it is shown in Figure 12, which supports this interpretation. The collectivity measured by the sum of the squared backward amplitudes is similar to the ${ }^{124}$ Xe case; $~ 0.6$ in both nuclei. Consequently the magnitudes of the interband $B(E 2)$ are also similar, although the $\Delta I=-1 B(E 2)$ is now larger than the $\Delta I=+1$ one because $\mathcal{Q}_{y} / \mathcal{Q}_{z}>0$, which is characteristic to small positive- $\gamma$ nuclei, see eq.(5.1). This trend of the triaxiality reflects the shape driving effect of the low- $\Omega$ aligned $\left(\pi h_{11 / 2}\right)^{2}$. The zigzag behaviour of the $M 1$ transitions are not pronounced at all in this case. This is because the $\mu_{z}$ amplitudes are very small. In addition, $M 1$ transitions is decreasing as a function of the rotational frequency, which is in contradiction to the prediction of the macroscopic formula.

An interesting feature of the data in this ${ }^{126} \mathrm{Ba}$ nucleus is that two branching ratios for decays from the members of the band under consideration, $T\left(17_{s \gamma}^{+} \rightarrow 16_{s}^{+}\right) / T\left(17_{s \gamma}^{+} \rightarrow 15_{s \gamma}^{+}\right)$ and $T\left(19_{s \gamma}^{+} \rightarrow 18_{s}^{+}\right) / T\left(19_{s \gamma}^{+} \rightarrow 17_{s \gamma}^{+}\right)$, where $s$ and $s \gamma$ stand for the $s$-band and the $\alpha=1 \gamma$-band excited on top of the $s$-band, respectively, were measured. Using the calculated values $B\left(E 2: I_{s \gamma} \rightarrow(I-1)_{s}\right)=0.044 \mathrm{e}^{2} \mathrm{~b}^{2}=12$ W.u. (c.f. Fig.12) and $B\left(E 2: I_{s \gamma} \rightarrow(I-2)_{s \gamma}\right) \simeq B\left(E 2:(I-1)_{s} \rightarrow(I-3)_{s}\right)=0.69 \mathrm{e}^{2} \mathrm{~b}^{2}=184$ W.u. at $\hbar \omega_{\text {rot }}=0.4 \mathrm{MeV}$ and measured $\gamma$ ray energies, the estimated branching ratio is $T(E 2$ : $\left.I_{s \gamma} \rightarrow(I-1)_{s}\right) / T\left(E 2: I_{s \gamma} \rightarrow(I-2)_{s \gamma}\right) \sim 0.05$, which is an order of magnitude smaller than the measured one. ${ }^{37)}$ This apparent contradiction between the data and the calculation can be solved by the specific property of the RPA solution in this case such that the $M 1$ transition is very strong, as is shown in Fig.13. Compare the $B(M 1)$ values with those of ${ }^{124}$ Xe in Fig.9.

As is pointed above the collectivity of the calculated solution in this case is more or less similar to that in ${ }^{124} \mathrm{Xe}$, which is a little bit weaker than that in ${ }^{182}$ Os. Actually, looking 
into the details of the microscopic structure of the RPA phonon, we have found appreciable amount of concentrations of the phonon amplitudes to the two-quasiparticle components with $\left(\pi h_{11 / 2}\right)^{2}$ in ${ }^{126} \mathrm{Ba}$ and with $\left(\nu h_{11 / 2}\right)^{2}$ in ${ }^{124} \mathrm{Xe}$, in contrast to the strong spread-over collectivity seen in the case of ${ }^{182}$ Os. The concentrations to the different orbits in ${ }^{126} \mathrm{Ba}$ and ${ }^{124} \mathrm{Xe}$ comes from the fact that the aligned quasiparticles in the vacuum configurations are protons in the former and neutrons in the latter, and then clearly explains why the $M 1$ transition is an order of magnitude stronger in ${ }^{126} \mathrm{Ba}$ than in ${ }^{124} \mathrm{Xe}$ (note also that the amount of concentration in the former is a little bit stronger than in the latter). In fact the value $B\left(M 1: I_{s \gamma} \rightarrow(I-1)_{s}\right)=0.28 \mu_{\mathrm{N}}^{2}=0.15 \mathrm{~W} . \mathrm{u}$. calculated at $\hbar \omega_{\text {rot }}=0.4 \mathrm{MeV}$ (c.f. Fig.13) explains the observed branching ratio very well. We think, therefore, these ${ }^{126} \mathrm{Ba}$ data are the first evidence of the rotationally induced "collective" $M 1$ transition between the $\gamma$ - and yrast bands in the sense that it has much larger $M 1$ transitions than E2 transitions though it is an even-even nucleus.

This $M 1$ matrix element on the other hand gives a possible explanation why the linking transition between the $s \gamma$ and $g \gamma$ bands in the $\alpha=1$ sector was not observed. The calculated reduced transition rates give $T(M 1)=0.23 \times 10^{13} s^{-1}$ and $T(E 2)=0.015 \times$ $10^{13} s^{-1}$ for $15_{s \gamma} \rightarrow 14_{s}\left(E_{\gamma}=0.781 \mathrm{MeV}\right)$ while $\left.T(E 2)<0.005 \times 10^{13} s^{-1}\right)$ for $15_{s \gamma} \rightarrow$ $13_{g \gamma}\left(E_{\gamma}=0.354 \mathrm{MeV}\right)$. The latter should be multiplied by a hindrance factor due to the band crossing in reality. Consequently the intensity of the $s \gamma$ band flows to the $s$ band rather than the $g \gamma$ band. The two mechanisms contribute to this results: the small transition energy in $I_{s \gamma} \rightarrow(I-2)_{g \gamma}$ and the large $M 1$ transitions in $I_{s \gamma} \rightarrow(I-1)_{s}$. In the case of ${ }^{182}$ Os with negative $\gamma$ deformation, however, this mechanism does not seem to apply because $E_{\gamma}\left(9_{s \gamma} \rightarrow 8_{s}\right)$ is also small and the $M 1$ transition are hindered.

\section{§5-5 Effects on properties of odd-A nuclei}

As has been discussed in the previous sections, the character of the lowest lying collective excitations in the $\alpha=1$ sector is $\gamma$-vibration like below the first band crossing while it sometimes acquires appreciable $K=1$ quadrupole strength and therefore becomes wobbling like above the band crossing. Rotational bands based on high- $j$ one-quasiparticle

*) This upper limit is estimated assuming a pure rotational E2 transitions as if no band crossing would exist. 
configurations in odd- $A$ nuclei decouple into a pair of $\Delta I=2$ sequences labeled by the different signature quantum number. The vibrational excitation mode under consideration in even-even nuclei affects signature-dependent properties of adjacent odd- $A$ nuclei through the particle-collective coupling. ${ }^{17)}$ We briefly summarize here the basic consequences of the dynamic coupling effects.

$B(E 2: I \rightarrow I-1)$ connecting these two sequences has been known to show signature dependence, i.e., zigzag behavior as a function of spin, which has been observed experimentally in some one-quasiparticle bands ${ }^{38,39)}$ before the band crossing. The theoretical studies has revealed that the signature dependence is brought about not only by the static ${ }^{40)}$ but also by the dynamic ${ }^{41-43)}$ triaxial deformations. These two effects usually contribute in opposite sign, and, for example, the effect of the dynamic one is partly canceled by that of the static one in ${ }^{157} \mathrm{Ho},{ }^{38)}$ while the latter is dominant in ${ }^{161} \mathrm{Dy} .{ }^{39)}$ This dynamic effect is nothing but the manifestation of the $K=2$ collectivity of the vibrational excitation considered in this paper and plays an important role to understand the electromagnetic properties of odd- $A$ nuclei.

Above the band crossing, i.e. in three-quasiparticle bands (e.g. $\left.\left(\pi h_{11 / 2}\right)^{1}\left(\nu i_{13 / 2}\right)^{2}\right)$ in odd- $A$ nuclei, the signature splitting of the excitation energy between the two sequences often becomes smaller than before the crossing and even becomes inverted in some cases; the so-called signature-inversion. This phenomenon has been explained in a similar way to that for odd-odd nuclei ${ }^{44-48)}$ as a result of positive $\gamma$ deformation. But this mechanism is not appropriate to explain the inversion in the three-quasiparticle bands which has been supposed to have negative- $\gamma$ deformation, for example, in the cases of ${ }^{165,167}{ }^{4{ }^{49}}{ }^{3}$ One of the present authors showed that the phase rule, eq.(5.1), results in stronger particlecollective coupling in the unfavoured $(I=j+$ odd $)$ sector than in the favored $(I=j+$ even $)$ sector in negative- $\gamma$ nuclei and consequently causes signature inversion. ${ }^{50)}$ In this model the effect of the static triaxiality is usually stronger and therefore the explanation in Refs. ${ }^{44-48)}$ consistently survives for positive- $\gamma$ nuclei. This example clearly shows, again, the importance of the collective vibrational modes in the high-spin spectroscopy. 


\section{$\S 6$. Concluding Remarks}

We have studied the nuclear wobbling motion from a microscopic viewpoint with paying special attention to the electromagnetic transition properties, especially $E 2$ and $M 1$, and to their implications in relation to the macroscopic Bohr-Mottelson model. Our basic stand point is the microscopic RPA theory suitable for high-spin states, which has

been proposed by Marshalek ${ }^{2,14)}$ and developed by the present authors. ${ }^{16,17)}$ In order to check the reliability of the microscopic framework, the rotational perturbation to the $E 2$ transitions between the $\gamma$-vibrational and the yrast bands in the low-spin region has been examined. The Bohr-Mottelson's generalized intensity relation are naturally comes out with the intrinsic transition moments which can now be calculated microscopically. The results of the calculations show both the absolute magnitude and the relative phase of these moments are well reproduced for some typical rare earth nuclei.

The strong rotational perturbation at high-spin states causes the structural change of the vacuum configuration, i.e. the quasiparticle alignments or the band-crossings. Accordingly the vibrational excitation modes are naturally expected to change their characters. In order to understand the character change, the interpretations of the observable quantities are necessary. For this purpose the microscopic wobbling model ${ }^{2)}$ based on the RPA theory has been reinvestigated in the light of the macroscopic wobbling model. ${ }^{1)}$ It has been shown that the expressions not only of excitation energy ${ }^{2)}$ but also of the electromagnetic transition rates, especially the $\Delta I= \pm 1 E 2$ transitions, can be cast into the form similar to those given in the macroscopic rotor model. Moreover, besides the collectivity of the RPA solutions, a criterion to interpret the wobbling motion has been clarified: The relative sign of the dynamic quadrupole asymmetries around the axes perpendicular to the main rotation axis, i.e. the transition amplitudes $\mathcal{Q}_{y}(n)$ and $\mathcal{Q}_{z}(n)$, eqs.(4.1) and (4.3), has to be the same as that of the static asymmetry around each axis, $\alpha_{y}$ and $\alpha_{z}$, eq.(3.8). Just as in the rotor model, the $E 2$ transitions depend also on the three moments of inertia which determine the excitation energy of the wobbling band at the same time. Therefore the wobbling motion reflects the three dimensional nature of the nuclear collective rotations.

The wobbling motion is an unique rotational motion in the sense that the rotation axis deviates from the inertia axis of the nuclear body. Recently, similar rotation scheme, called 
"tilted axis cranking", ${ }^{51)}$ is proposed and has been applied to the study of the rotational bands with no-signature splitting. Although both rotations are of the three dimensional nature, they are conceptually different: the wobbling motion is not the stationary motion and the angular momentum and the angular frequency vectors draw different trajectories in the body-fixed frame, while the tiled cranking is the stationary rotation and then the angular momentum and angular frequency vectors are parallel each other with fixed direction with respect to the body-fixed frame. In fact, these two vector have a definite relationship in the wobbling motion, which is nothing but determined by the three moments of inertia (generally the moment of inertia tensor ${ }^{52)}$ ). Moreover, when quantized spectra are considered, the tilted axis cranking gives a description for an isolated band just like the usual cranking does, but the wobbling motion as a whole corresponds to a multiple band structure, although we concentrated mainly on the first excited band in this paper.

Some examples of the realistic calculations are also presented in this paper. The sign relation mentioned above leads characteristic zigzag behaviours in the $E 2$ and $M 1$ transition probabilities as functions of the rotational frequency, depending on equilibrium values of $\gamma$ deformation in the $\left(\epsilon_{2}, \gamma\right)$-plane. This is quite analogous to the case of the $B(M 1)$ between the signature partner bands in odd- $A$ nuclei. An important merit of the microscopic RPA formalism is that the three moments of inertia can be easily calculated, which is highly nontrivial. Their behaviour in three sectors of the $\left(\epsilon_{2}, \gamma\right)$-plane has been calculated in a rather simplified manner with neglecting selfconsistency of the mean-field potential. The results clearly show that their $\gamma$ dependence is neither irrotational nor rigid-body like.

As a candidate of the nuclear wobbling motion which might be identified experimentally, we have also investigated the high-spin continuations of the so-called $\gamma$-vibrational bands with odd-spin in ${ }^{182} \mathrm{Os},{ }^{124} \mathrm{Xe}$, and ${ }^{126} \mathrm{Ba}$. The selfconsistent triaxiality deformation is somewhat different in each nucleus, and therefore the characteristic features of the collective vibrational modes are shown also to be different. Properties of the second-lowest RPA eigenmode, which is in some cases also rather collective but does not satisfy the criterion of the wobbling motion, are also discussed.

Throughout this paper the $M 1$ transition between the wobbling and yrast bands has 
also been kept in scope. It should, however, be noticed that the expression of the $B(M 1)$ in the rotor model are not justified from the microscopic viewpoint. This is because the individual property of quasiparticle orbits are essential to understand the $M 1$ transitions, which is not taken into account in the macroscopic model, in contrast to the case of the $E 2$ transitions, where the geometrical shape of the nucleus as a whole is mainly responsible. Although the $M 1$ transitions from the quadrupole vibrational band are generally expected to be small at low-spin, it has been shown that this is not always the case at higher-spin region, where the rotation-aligned quasiparticles present in the vacuum. Note that the $M 1$ transition is only possible if there exist the rotationally induced $K$ mixing in the RPA phonon amplitudes. As a conspicuous example the high-spin continuation of the odd-spin $\gamma$-vibrational band in ${ }^{126} \mathrm{Ba}$ is predicted to have much stronger $M 1$ transition probability than the $\Delta I= \pm 1 E 2$, which is consistent with the observed branching ratio. This strong $M 1$ transition, on the other hand, gives a possible reason why the $\Delta I=-2$ transition that links above and below the band crossing in the odd-spin $\gamma$ band has not been observed.

It is worthwhile to stress the importance of the collective vibrational motions of either the wobbling like or of the $\gamma$-vibrational character for the understanding the electromagnetic property of the odd- $A$ nuclei. The characteristic property of the vibrational modes reflect strongly on the transition rates through the particle-vibration coupling effects, and therefore the study of the odd- $A$ nuclei gives a valuable testing ground to clarify the character change of the vibrational mode predicted in the present work. We hope that new generation of the large array of the crystal ball will provide us more detailed information of the electromagnetic transitions, which is necessary to confirm the predictions and to identify the nuclear wobbling motion if they exist.

\section{Acknowledgements}

Discussions with R. Wyss are greatly acknowledged. Early stage of this work has been done as a part of the research project on Nonlinear Dynamics of Nuclear Collective Motions, which were organized at the Yukawa Institute, Kyoto, in 1988-1992. We had stimulating discussions with all the members. This work is financially supported in part by the Grant-in-Aid for Scientific Research from Ministry of Education, Science and Culture (No. 06234208). 


\section{Table 1.}

The first order coefficient of the rotational frequency dependence calculated by the RPA theory, $a_{\gamma}$ in eq.(2.5) and the extracted intensity relation parameters, $\mathcal{Q}_{t}$ and $q$ in eq. $(2.9 \mathrm{~b})$. Here $(g)$ and $(\gamma)$ attached to the theoretical $q$ values mean that they are obtained by eq.(2.9b) using experimental moment of inertia of the ground band $\left(\mathcal{J} \equiv \mathcal{J}_{g}\right)$ and of the $\gamma$ band $\left(\mathcal{J} \equiv \mathcal{J}_{\gamma}\right)$.

\begin{tabular}{ccccccc}
\hline Nucl. & $a_{\gamma} / \hbar\left[\mathrm{MeV}^{-1}\right]$ & $\mathcal{Q}_{t}^{(t h)}[\mathrm{eb}]$ & $\mathcal{Q}_{t}^{(e x p)}[\mathrm{eb}]$ & $q^{(t h)}(g)$ & $q^{(t h)}(\gamma)$ & $q^{(e x p)}$ \\
\hline${ }^{162} \mathrm{Dy}$ & 2.0 & 0.37 & $0.35^{1)}$ & 0.027 & 0.024 & - \\
${ }^{164} \mathrm{Dy}$ & 1.9 & 0.38 & $0.34^{2)}$ & 0.023 & 0.020 & $0.021^{2)}$ \\
${ }^{164} \mathrm{Er}$ & 2.0 & 0.41 & $0.38^{3)}$ & 0.031 & 0.027 & - \\
${ }^{166} \mathrm{Er}$ & 1.8 & 0.41 & $0.42^{4)}$ & 0.024 & 0.021 & $0.022^{4)}$ \\
${ }^{168} \mathrm{Er}$ & 1.3 & 0.42 & $0.37^{5)}$ & 0.017 & 0.015 & $0.018^{5)}$ \\
\hline
\end{tabular}

1) Extracted from the $B\left(E 2: 0_{g}^{+} \rightarrow 2_{\gamma}^{+}\right){ }^{53)}$

2) Extracted from the $\chi^{2}$ fitting of the matrix elements $\mathcal{M}(E 2 ; \Delta I=0,-2)_{\gamma \rightarrow g}$ $\left(I_{\gamma}=2,4, \ldots, 10\right)$ obtained by the Coulomb excitation experiment of Ref.. ${ }^{54)}$ There is an appreciable scattering of data from the relation, eq.(2.9a). The weights inversely propotional to the square of error bars of data are used for the $\chi^{2}$ fitting.

3) Extracted from the $B\left(E 2: 0_{g}^{+} \rightarrow 2_{\gamma}^{+}\right)^{55)}$

4) From Ref.. ${ }^{19)}$

5) Extracted from the $\chi^{2}$ fitting of the matrix elements $\mathcal{M}(E 2 ; \Delta I=$ $0, \pm 1, \pm 2)_{\gamma \rightarrow g}\left(I_{\gamma}=2,3, \ldots, 8\right)$ obtained by the Coulomb excitation experiment in Ref. ${ }^{56)}$ just in the same way as 2 ). 


\section{Figure Captions}

Fig.1 The electric E2 transition amplitudes, eq.(2.4), in ${ }^{164}$ Er microscopically calculated by the RPA formalism as functions of the rotational frequency.

Fig.2 Schematic figure depicting the analogy of the $\Delta I= \pm 1 \quad E 2$ transitions between the yrast and the wobbling bands in even-even nuclei with the $M 1$ transitions between the signature partner bands in odd nuclei.

Fig.3 Schematic figure depicting the relation between the triaxiality of the mean-field and the $\Delta I= \pm 1 E 2$ transitions from the wobbling band to the vacuum band. The $\left(\epsilon_{2}, \gamma\right)$ plane is classified into three regions, for which characteristic zigzag behaviours of $B(E 2)_{\Delta I= \pm 1}$ are shown in the middle panels. The transitions with stronger $B(E 2)$ 's are marked in the spectra (right panels).

Fig.4 The $E 2$ transition amplitudes, $\mathcal{Q}_{y}$ and $\mathcal{Q}_{z}$ (left panel) and $\Delta I= \pm 1 B(E 2)$ (right panel) for the lowest RPA solutions with signature $\alpha=1$ as functions of the rotational frequency in ${ }^{182}$ Os. The calculational procedures are the same as those in $\S 2$ but the mean-field parameters are fixed as $\epsilon_{2}=0.21$, and $\gamma=-18^{\circ}$, and $\Delta_{\nu}=0.74, \Delta_{\pi}=0.98$ $\mathrm{MeV}$, which are appropriate for the s-bands in ${ }^{182} \mathrm{Os}^{25)}$ As a reference the Weisskopf unit of $B(E 2)$ in this case is $0.0061 \mathrm{e}^{2} \mathrm{~b}^{2}$.

Fig.5 The effective moment of inertia as functions of the triaxial parameter $\gamma$ at $\hbar \omega_{\text {rot }}=0.25$ $\mathrm{MeV}$. The results for $-120^{\circ}<\gamma<-60^{\circ}$ in ${ }^{176} \mathrm{Hf}$, for $-60^{\circ}<\gamma<0^{\circ}$ in ${ }^{182} \mathrm{Os}$ and for $0^{\circ}<\gamma<60^{\circ}$ in ${ }^{148} \mathrm{Gd}$ are gathered in one panel. The mean-field parameters except $\gamma$ are fixed as $\epsilon_{2}=0.27$ and 0.22 for ${ }^{176} \mathrm{Hf}$ and ${ }^{148} \mathrm{Gd}$, respectively, and $\Delta_{\nu}=0.6$, $\Delta_{\pi}=0.6 \mathrm{MeV}$ for both nuclei. The same parameters are used for ${ }^{182} \mathrm{Os}$ as Fig.4. Note that ${ }^{176} \mathrm{Hf}$ and ${ }^{148} \mathrm{Gd}$ are known to be the nuclei with prolate and oblate non-collective rotations so that the selfconsistent $\gamma$ deformations in these nuclei are $\gamma=-120^{\circ}$ and $60^{\circ}$, respectively. The diabatic configurations of the vacuum states are chosen to be two-quasineutron four-quasiproton states for ${ }^{176} \mathrm{Hf}$, two-quasineutron states ( $s$-band) for ${ }^{182} \mathrm{Os}$, and two-quasineutron and two-quasiproton states for ${ }^{148} \mathrm{Gd}$. For ${ }^{176} \mathrm{Hf}$ and ${ }^{148} \mathrm{Gd}$, these configurations correspond to the high- $K$ states with spin $20^{+}$and $18^{+} \hbar$, respectively, which are the yrast with $(\pi, \alpha)=(+, 0)$ for both neutron and proton at their selfconsistent deformations. Unfortunately these high- $K$ states do not coincide with any of the observed yrast isomers.

Fig.6 Routhians for the lowest lying state in the $\alpha=1$ sector as functions of the rotational frequency in ${ }^{124} \mathrm{Xe}$. The calculated one is shown by the solid line and the experimental one, which is the routhian of the odd-spin members of the so-called $\gamma$ band, by the dashed line, and the observed yrast band by the short-dashed line. Experimental routhians are shown with respect to a reference band with $\mathcal{J}_{0}=15.0 \hbar^{2} / \mathrm{MeV}, \mathcal{J}_{1}=$ $25.0 \hbar^{4} / \mathrm{MeV}^{3}, e_{s}=3.03 \mathrm{MeV}$ and $i_{s}=7.14 \hbar$. Parameters used for the calculation 
are $\epsilon_{2}=0.19, \gamma=-45^{\circ}$ and $\Delta_{\nu}=0.9, \Delta_{\pi}=1.1 \mathrm{MeV}$.

Fig.7 Calculated effective moments of inertia of the lowest lying state in the $\alpha=1$ sector in ${ }^{124} \mathrm{Xe}$ as functions of the rotational frequency. Parameters used are the same as in Fig.6.

Fig.8 The $E 2$ transition amplitudes, $\mathcal{Q}_{y}$ and $\mathcal{Q}_{z}$ (left panel) and $\Delta I= \pm 1 B(E 2)$ (right panel) for the lowest RPA solutions with signature $\alpha=1$ as functions of the rotational frequency in ${ }^{124}$ Xe. Parameters used are the same as in Fig.6. As a reference the Weisskopf unit of $B(E 2)$ in this case is $0.0037 \mathrm{e}^{2} \mathrm{~b}^{2}$.

Fig.9 The $M 1$ transition amplitudes, $i \mu_{y}$ and $\mu_{z}$ (left panel) and $B(M 1)$ (right panel) for the lowest RPA solutions with signature $\alpha=1$ as functions of the rotational frequency in ${ }^{124}$ Xe. Parameters used are the same as in Fig.6. As a reference the Weisskopf unit of $B(M 1)$ is $1.79 \mu_{N}^{2}$. Note that the relative phase between the $M 1$ amplitudes and the $E 2$ amplitudes (Fig.8) is meaningful and gives the sign of the E2/M1 mixing ratio.

Fig.10 Excitation energies (left panel) and the $E 2$ transition amplitudes (right panel) of the lowest and second-lowest states in the $\alpha=1$ sector in ${ }^{124}$ Xe calculated at $\hbar \omega_{\text {rot }}=0.3$ $\mathrm{MeV}$ as functions of $\gamma$. Parameters used for the calculation except $\gamma$ are the same as Fig.6.

Fig.11 The same as Fig.6 but for ${ }^{126}$ Ba. Parameters used for the calculation are $\epsilon_{2}=0.24$, $\gamma=0$, and selfconsistently calculated pairing gaps are $1.16 \geq \Delta_{\nu} \geq 1.03 \mathrm{MeV}$ and $0.76 \geq \Delta_{\pi} \geq 0.71 \mathrm{MeV}$ for $0.4 \leq \hbar \omega_{\text {rot }} \leq 0.5 \mathrm{MeV}$. A reference band with $\mathcal{J}_{0}=$ $14.0 \hbar^{2} / \mathrm{MeV}, \mathcal{J}_{1}=31.0 \hbar^{4} / \mathrm{MeV}^{3}, e_{s}=3.00 \mathrm{MeV}$ and $i_{s}=7.64 \hbar$ is used here. Note that the $\gamma$-ray which links above and below the band crossing has not been observed (see the text).

Fig.12 The same as Fig. 8 but for ${ }^{126}$ Ba. Parameters used are the same as in Fig.11. As a reference the Weisskopf unit of $B(E 2)$ in this case is $0.0038 \mathrm{e}^{2} \mathrm{~b}^{2}$.

Fig.13 The same as Fig.9 but for ${ }^{126}$ Ba. Parameters used are the same as in Fig.11. 


\section{References}

1. A. Bohr and B. R. Mottelson, Nuclear Structure, Vol. II, (Benjamin, New York, 1975), Chap. 4, p.190 ff.

2. E. R. Marshalek, Nucl. Phys. A331 (1979), 429.

3. D. R. Bes and J. Kurchan, The Treatment of Collective Coordinates in Many-Body Systems, World Scientific Lecture Notes in Physics, Vol.34, 1990, World Scientific Publishing Co.

4. A. K. Kerman and N. Onishi, Nucl. Phys. A361 (1981), 179.

5. N. Onishi, Nucl. Phys. A456 (1986), 390.

6. K. Kaneko, Phys. Rev. 45 (1992), 2754; Phys. Rev. 49 (1994), 3014.

7. I. N. Mikhailov and D. Janssen, Phys. Lett. B72 (1978), 303.

8. D. Janssen and I. N. Mikhailov, Nucl. Phys. A318 (1979), 390.

9. V. G. Zelevinsky, Nucl. Phys. A344 (1980), 109.

10. J. Kurchan, D. R. Bes and S. Cruz Barrios, Nucl. Phys. A509 (1990), 306.

11. Y. R. Shimizu, T. Kisaka and M. Matsuzaki, Soryushiron Kenkyu (Kyoto) 81 (1990), No.61, F182.

12. Y. R. Shimizu and M. Matsuzaki, in Proceedings of the International Conference on Nuclear Structure at High Angular Momentum, May 18-21, 1992, Ottawa, AECL-10613, pp.278-282.

13. Y. R. Shimizu and M. Matsuzaki, in Proceedings of the International Conference on Physics from Large Gamma-ray Detector Arrays, August 2-6, 1994, Berkeley, California, to be published.

14. E. R. Marshalek, Phys. Rev. C11 (1975), 1426; Nucl. Phys. A266 (1976), 317.

15. J. L. Egido, H. J. Mang and P. Ring, Nucl. Phys. A339 (1980), 390.

16. Y. R. Shimizu and K. Matsuyanagi, Prog. Theor. Phys. 70 (1983), 144; 72 (1984), 799.

17. M. Matsuzaki, Y. R. Shimizu and K. Matsuyanagi, Prog. Theor. Phys. 79 (1988), 836.

18. F. Dönau, Nucl. Phys. A471 (1987), 469.

19. A. Bohr and B. R. Mottelson, Nuclear Structure, Vol. II, (Benjamin, New York, 1975), Chap. 4, p.158 ff.

20. M. Baranger and K. Kumar, Nucl. Phys. A110 (1968), 490.

21. H. Sakamoto, Nucl. Phys. A557 (1992), 583c.

22. K. E. G. Löbner, M. Vetter and V. Hönig, Nuclear Data Tables, A7 (1970), 495.

23. L. D. Landau and E. M. Lifshitz, Mechanics, 3-rd ed., (Pergamon, London, 1976), $\S 37$.

24. M. M. Villard, Ph. Hubert and R. J. Liotta, Physica Scripta, 26 (1982), 201. 
25. M. Matsuzaki, Nucl. Phys. A509 (1990), 269.

26. C. G. Andersson et. al., Nucl. Phys. A361 (1981), 147.

27. J. Skalski, Nucl. Phys. A473 (1987), 40.

28. H. Kurasawa, Prog. Theor. Phys. 64 (1980), 2055; 66 (1981), 1317; 68 (1982), 1594.

29. Y. R. Shimizu, J. D. Garrett, R. A. Broglia, M. Gallardo, and E. Vigezzi, Rev. Mod. Phys. 61 (1989), 131.

30. G. B. Hagemann and I. Hamamoto, Phys. Rev. C40 (1989), 2862.

31. P. Chowdhury et. al.,Nucl. Phys. A485 (1988), 136.

32. W. Gast et al., Z.Phys. A318 (1984), 123.

33. G. Puddu, O. Scholten and T. Otsuka, Nucl. Phys. A348 (1980), 109.

34. S. Törmänen et al., Nucl. Phys. A572 (1994), 417.

35. R. Wyss et al., Nucl. Phys. A505 (1989), 337.

36. A. Bohr and B. R. Mottelson, Nuclear Structure, Vol. I, (Benjamin, New York, 1969), Chap. 3-3b.

37. D. Ward et al., Nucl. Phys. A529 (1991), 315.

38. D. C. Radford et al., Nucl. Phys. A545 (1992), 665.

39. M. Oshima et al., Phys. Rev. C37 (1988), 2578.

40. I. Hamamoto and B. R. Mottelson, Phys. Lett. B132 (1983), 7.

41. A. Ikeda, Nucl. Phys. A439 (1985), 317.

42. N. Onishi, I. Hamamoto, S. Åberg and A. Ikeda, Nucl. Phys. A452 (1986), 71.

43. M. Matsuzaki, Nucl. Phys. A491 (1989), 433; ibid A519 (1990), 548.

44. R. Bengtsson et al., Nucl. Phys. A415 (1985), 189.

45. I. Hamamoto and B. Mottelson, Phys. Lett. B127 (1983), 281.

46. A. Ikeda and S. Åberg, Nucl. Phys. A480 (1988), 85.

47. N. Onishi and N. Tajima, Prog. Theor. Phys. 80 (1988), 130.

48. M. Matsuzaki, Nucl. Phys. A504 (1989), 456.

49. C. -H. Yu et al., Nucl. Phys. A511 (1990), 157.

50. M. Matsuzaki, Phys. Rev. C46 (1992), 1548.

51. S. Frauendorf, Nucl. Phys. A557 (1993), 259c.

52. A. L. Goodman, Phys. Rev. C45 (1992), 1649.

53. R. G. Helmer, Nuclear Data Sheets 64 (1991), 79.

54. H. Kusakari et. al., JAERI Tandem Annual Report 1992, 125.

55. R. M. Ronningen et. al., Phys. Rev. C26 (1982), 97.

56. B. Kotliński et. al., Nucl. Phys. A517 (1990), 365. 
This figure "fig1-1.png" is available in "png" format from: http://arxiv.org/ps/nucl-th/9409016v1 
This figure "fig2-1.png" is available in "png" format from: http://arxiv.org/ps/nucl-th/9409016v1 
This figure "fig3-1.png" is available in "png" format from: http://arxiv.org/ps/nucl-th/9409016v1 
This figure "fig4-1.png" is available in "png" format from: http://arxiv.org/ps/nucl-th/9409016v1 
This figure "fig1-2.png" is available in "png" format from: http://arxiv.org/ps/nucl-th/9409016v1 
This figure "fig2-2.png" is available in "png" format from: http://arxiv.org/ps/nucl-th/9409016v1 
This figure "fig3-2.png" is available in "png" format from: http://arxiv.org/ps/nucl-th/9409016v1 
This figure "fig4-2.png" is available in "png" format from: http://arxiv.org/ps/nucl-th/9409016v1 
This figure "fig1-3.png" is available in "png" format from: http://arxiv.org/ps/nucl-th/9409016v1 
This figure "fig2-3.png" is available in "png" format from: http://arxiv.org/ps/nucl-th/9409016v1 
This figure "fig3-3.png" is available in "png" format from: http://arxiv.org/ps/nucl-th/9409016v1 
This figure "fig4-3.png" is available in "png" format from: http://arxiv.org/ps/nucl-th/9409016v1 
This figure "fig4-4.png" is available in "png" format from: http://arxiv.org/ps/nucl-th/9409016v1 